SUBMitTed TO APJ

Preprint typeset using $\mathrm{LAT}_{\mathrm{E}} \mathrm{X}$ style emulateapj v. 12/16/11

\title{
THE PIPER SURVEY: I. AN INITIAL LOOK AT THE INTERGALACTIC GLOBULAR CLUSTER POPULATION IN THE PERSEUS CLUSTER
}

\author{
William E. HARRIS \\ Department of Physics \& Astronomy \\ McMaster University \\ Hamilton ON L8S 4M1, Canada \\ RACHEL A. BROWN \\ Department of Physics \& Astronomy \\ McMaster University \\ Hamilton ON L8S 4M1, Canada \\ PATRICK R. DurRell \\ Department of Physics and Astronomy \\ Youngstown State University \\ Youngstown $\mathrm{OH} 44555$, USA \\ Aaron J. RomanowsKy \\ Department of Physics \& Astronomy, San José State University \\ One Washington Square, San José CA 95192, USA \\ and University of California Observatories, 701156 High Street, Santa Cruz CA 95064, USA \\ JOHN BLAKESLEE \\ National Research Council of Canada \\ Herzberg Astronomy and Astrophysics Research Centre \\ Victoria BC, Canada \\ JEAN BRODIE \\ University of California Observatories \\ 1156 High Street, Santa Cruz CA 95064, USA \\ STEVEN JANSSENS \\ Department of Astronomy and Astrophysics \\ University of Toronto, 50 St. George St, Toronto ON M5S 3H4, Canada
}

THORSTEN LiSKER

Astronomisches Rechen-Institut, Zentrum für Astronomie der Universität Heidelberg, Mönchhofstraße 12-14, 69120 Heidelberg, Germany

SAKURAKO OKAMOTO

Subaru Telescope, National Astronomical Observatory of Japan, 650 North Aohoku Place, Hilo, HI 96720, U.S.A.

National Astronomical Observatory of Japan, Osawa 2-21-1, Mitaka, Tokyo, 181-8588, JAPAN and

The Graduate University for Advanced Studies, Osawa 2-21-1, Mitaka, Tokyo 181-8588, JAPAN

CAROLin Wittmann

Astronomisches Rechen-Institut, Zentrum für Astronomie der Universität Heidelberg, Mönchhofstraße 12-14, 69120 Heidelberg, Germany (Dated: January 20, 2020)

$$
\text { submitted to ApJ }
$$

\section{ABSTRACT}

We describe the goals and first results of a Program for Imaging of the PERseus cluster of galaxies (PIPER). The first phase of the program builds on imaging of fields obtained with the Hubble Space Telescope (HST) ACS/WFC and WFC3/UVIS cameras. Our PIPER target fields with HST include major early-type galaxies including the active central giant NGC 1275; known Ultra-Diffuse Galaxies; and the Intracluster Medium. The resulting two-color photometry in F475W and F814W reaches deep enough to resolve and measure the globular cluster (GC) populations in the Perseus member galaxies. Here we present initial results for eight pairs of outer fields that confirm the presence of Intergalactic GCs (IGCs) in fields as distant as $740 \mathrm{kpc}$ from the Perseus center (40\% of the virial radius of the cluster). Roughly $90 \%$ of these IGCs are identifiably blue (metal-poor) but there is a clear trace of a 
red (metal-rich) component as well, even at these very remote distances.

Keywords: galaxies: formation — galaxies: star clusters — globular clusters: general

\section{INTRODUCTION}

Perseus (Abell 426) at $d=75 \mathrm{Mpc}$ offers a rich and fascinating laboratory for galaxy evolution, but it has not yet gained the level of attention that has been given, for example, to Virgo or Coma. Perseus has a velocity dispersion in the range $\sigma_{v} \simeq 1000-1300 \mathrm{~km} \mathrm{~s}^{-1}$, among the highest of clusters in the local universe (Kent \& Sargent 1983. Struble \& Rood 1991; Girardi et al. 1996; Weinmann et al. 2011); for comparison, the Coma cluster has $\sigma_{v}=1100 \mathrm{~km} \mathrm{~s}^{-1}$ Colless \& Dunn 1996). The total mass of Perseus is almost $10^{15} M_{\odot}$ (Girardi et al. 1998 Simionescu et al. 2011). Within the bounds of the cluster is a vast halo of X-ray gas and dark matter with virial radius $r_{200}=1.8 \mathrm{Mpc}$ (Simionescu et al. 2011), comparable to the most gas-rich and populous galaxy clusters known (e.g. Zhao et al. 2013, Loewenstein 1994, Main et al. 2017).

Like Virgo and Coma, Perseus contains many large early-type galaxies, the most notable of which is the central supergiant NGC 1275 (= 3C48 = Perseus A), which sits at the center of the X-ray gas and the dynamical center of the cluster. NGC 1275 is perhaps the most extreme case in the local universe where we see the ongoing growth of a Brightest Cluster Galaxy (BCG) complete with cooling flows and feedback, though globally its stellar mass is dominated by an old, passive population typical of a giant elliptical. Within NGC 1275 is a spectacular web of $\mathrm{H} \alpha$ filaments extending to more than $30 \mathrm{kpc}$ from the galaxy center, which itself contains $\sim 10^{11} M_{\odot}$ of molecular gas and extended regions of star formation (e.g. Fabian et al. 2011; Matsushita et al.|2013. Canning et al. 2014).

In this paper, we describe a Program for Imaging of PERseus (called PIPER) currently underway that is intended to attack four different program goals through the use of globular cluster (GC) populations.

(1) In recent years it has become clear that rich clusters of galaxies are hosts for large numbers of Ultra-Diffuse Galaxies (UDGs), currently a focus of considerable interest: recent observational work includes van Dokkum et al. (2015a b); Mihos et al. (2015); Koda et al. (2015); Martínez-Delgado et al. (2016); Román \& Trujillo (2017); Papastergis et al. (2017); Amorisco et al. (2018); Janssens et al. (2017), now accompanied by a growing literature on theoretical modelling (e.g. Rong et al. 2017; Chan et al. 2018; Jiang et al. 2019; Carleton et al. 2019; Martin et al. 2019). Perseus is already known to hold many UDG candidates (Wittmann et al. 2017), giving us the chance to improve our understanding of their demographics. Some UDGs in turn seem to have remarkably populous systems of GCs relative to their low luminosities (Peng \& Lim 2016, Beasley \& Trujillo 2016, van Dokkum et al. 2016, 2018), indicating that they have massive dark halos and extremely high mass-to-light ratios (Harris et al. 2017b), while others are quite GC-poor (Lim et al. 2018; Amorisco et al. 2018). No highly consistent pattern has yet emerged, and at least some of these extreme galax-

harris@physics.mcmaster.ca ies may violate the near-constant ratio of GC system mass to halo (virial) mass obeyed by more luminous galaxies (Hudson et al. 2014; Durrell et al. 2014, Harris et al. 2017b). Said differently, for the lowest-luminosity galaxies including the UDGs, the scatter around either the $M_{G C S}-M_{v i r}$ relation or around the stellar-to-halomass relation (SHMR) may increase significantly; for discussion of these issues, see the papers cited above and (Toloba et al. 2018, El-Badry et al. 2018, Amorisco et al. 2018, Lim et al. 2018, Forbes et al. 2018, Prole et al. 2019, Burkert \& Forbes 2019).

(2) As a rich and dynamically active cluster, Perseus should also have stellar Intra-Cluster Light (ICL) built from disrupted or stripped member galaxies (e.g. Burke et al. 2012, Ramos et al. 2015, Ramos-Almendares et al. 2018). In such clusters the ICL can make up typically 10$30 \%$ of the total stellar mass. Because the ICL is actively growing particularly since $z=1$, the sheer amount of ICL and its degree of substructure (clumpiness and tidal streams) probe the dynamical state of the entire cluster. But the stellar ICL is extremely diffuse and difficult to map out with conventional surface-brightness photometry over a field as wide as the entire field of Perseus, whose virial radius is near $\sim 1.5^{\circ}$. For such a low signal, a more effective tracer is one for which the background 'noise' can be reduced to near-zero levels. As will be shown below, GCs fit this bill beautifully: they are individually luminous, easy to isolate, and can be reached by HST for galaxies out to $\sim 250 \mathrm{Mpc}$ or more (Harris et al. 2016).

Substantial numbers of intergalactic globular clusters (IGCs) have been detected in other large clusters including Virgo (Lee et al. 2010; Durrell et al. 2014, Ko et al. 2017; Longobardi et al. 2018), Coma (Peng et al. 2011; Madrid et al. 2018), A1185 (West et al. 2011), and A1689 (Alamo-Martínez \& Blakeslee 2017). Perhaps most importantly, multiband photometry also automatically yields their metallicity distribution function (MDF) since, for old ( $\gtrsim 3$ Gyr) clusters, GC color is a monotonic function of $[\mathrm{Fe} / \mathrm{H}]$ and is quite insensitive to age (e.g. Peng et al. 2006). The extremely faint, diffuse, integrated stellar light cannot provide this level of insight at large distances from galaxy center.

Harris \& Mulholland (2017, hereafter HM17) carried out a preliminary investigation of the GC populations within Perseus using MAST Archival ACS and WFPC2 fields. They found evidence for IGCs, albeit with large uncertainties. In and near the Perseus core, any IGC component can be easily confused with (or dominated by) the rich GC systems around the giant early-type galaxies, while in the remoter outskirts of Perseus, their conclusions relied on only a handful of shallower WFPC2 images.

(3) The Perseus core has several large early-type galaxies (ETGs), particularly NGC 1275, NGC 1272, and NGC 1278. Since their projected separations are $\lesssim 100 \mathrm{kpc}$, the total GC population in the core region will be a mutually overlapping combination of these three major galaxies, plus some smaller Perseus members, plus the IGCs 


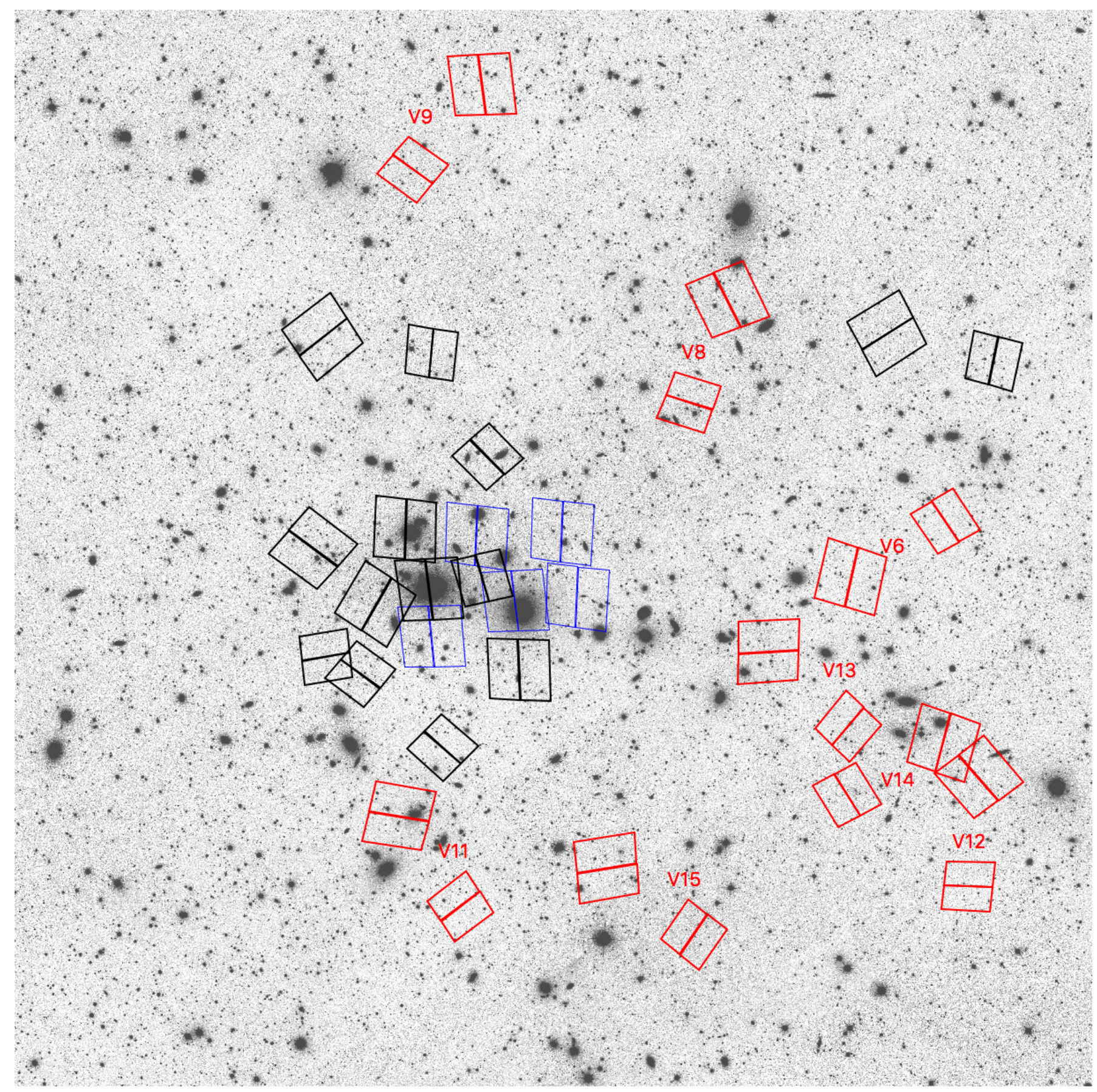

Figure 1. Image of the central region of the Perseus cluster generated from an SDSS image stack in $r$. North is at top, East at left and the field shown is $60^{\prime}=1300 \mathrm{kpc}$ across. The locations of the HST pointings used in this study are shown as pairs of rectangles (ACS the bigger, WFC3 the smaller in each pair). NGC 1275, at the center of Perseus, is left of center. The pointings specifically discussed in this paper are marked in red and labelled with their Visit numbers from Table 1. The five thin blue single ACS fields near the center show previous fields from the MAST archive, not used in the present paper but part of our larger PIPER analysis to come.

(which should follow to first order the cluster potential well). Given deep photometry with appropriate field coverage of this central region, a simultaneous solution for all these components can be performed. NGC 1275 is uniquely interesting because GC systems have never been explored in a galaxy with such extreme, high-activity conditions. If, for example, its GCS turns out to have high specific frequency (number of GCs per unit galaxy luminosity) as is the usual case for BCGs (Harris et al. 2017c), it would support the view that the current spec- tacular AGN and star-forming activity including young $(<1 \mathrm{Gyr})$ star clusters in its inner $20 \mathrm{kpc}$ (Carlson et al. 1998 Canning et al. 2010, 2014, Lim et al. 2019) is only an add-on to a dominant early formation epoch.

(4) At the opposite end of the dwarf-galaxy structural scale from the UDGs are the Ultra-Compact Dwarfs (UCDs). Their characteristic luminosities $\gtrsim 10^{7} L_{\odot}$ and radii $r_{h} \gtrsim 10 \mathrm{pc}$ distinguish them from all but the largest and most luminous GCs. These dense stellar systems may be a mixed population, either remnant nuclei of 
Table 1

TARGET FieLDS

\begin{tabular}{|c|c|c|c|c|c|c|c|c|}
\hline MAST Field Label & Visit & RA & Dec & $R^{\prime}$ & $A_{I}$ & $t_{B}, t_{I}(\mathrm{ACS})$ & $t_{B}, t_{I}(\mathrm{WFC} 3)$ & Target Galaxies \\
\hline NGC1275-F1 & V1 & 03:19:49.6 & $+41: 30: 36.0$ & 0.29 & 0.245 & 2436,2325 & 2535,2535 & NGC 1275 \\
\hline NGC1275-F2 & $\mathrm{V} 2$ & 03:20:03.5 & $+41: 29: 39.4$ & 3.06 & 0.245 & 2436,2325 & 2535,2535 & Perseus core \\
\hline NGC1275-F3 & V3 & $03: 19: 54.5$ & $+41: 33: 54.5$ & 3.42 & 0.247 & 2577,2429 & 2659,2772 & Perseus core \\
\hline NGC1275-F4 & V4 & $03: 19: 23.1$ & $+41: 26: 09.3$ & 6.53 & 0.242 & 2436,2325 & 2535,2535 & Perseus core \\
\hline NGC1275-F5 & V5 & $03: 20: 23.8$ & $+41: 32: 43.1$ & 6.97 & 0.249 & 2577,2429 & 2659,2772 & NGC 1278 \\
\hline PERSEUS-UDG01 & V6 & $03: 17: 16.0$ & $+41: 34: 10.1$ & 28.70 & 0.244 & 2424,2271 & 2533,2653 & WUDG $5,8,13,14$ \\
\hline PERSEUS-UDG02 & V7 & $03: 17: 00.3$ & $+41: 42: 58.9$ & 33.74 & 0.227 & 2436,2105 & 2604,2640 & RUDG $5,6,84$ \\
\hline PERSEUS-UDG03 & V8 & $03: 18: 30.2$ & $+41: 41: 07.1$ & 17.93 & 0.256 & 2436,2359 & 2604,2640 & WUDG $28,29,33,35,36,40,41$, RUDG 25 \\
\hline PERSEUS-UDG04 & V9 & $03: 19: 54.1$ & $+41: 54: 01.7$ & 23.35 & 0.276 & 2436,2359 & 2604,2640 & RUDG 23,60 \\
\hline PERSEUS-UDG05 & V10 & $03: 19: 50.2$ & $+41: 43: 19.3$ & 12.63 & 0.252 & 2436,2539 & 2604,2640 & WUDG 83,84, RUDG 21,27 \\
\hline PERSEUS-UDG06 & V11 & $03: 19: 39.9$ & $+41: 13: 07.6$ & 17.64 & 0.228 & 2402,2271 & 2533,2653 & WUDG $79,80,88,89$ \\
\hline PERSEUS-UDG07 & V12 & 03:17:09.0 & $+41: 14: 06.4$ & 34.11 & 0.227 & 2424,2271 & 2533,2653 & WUDG $1,2,7$, RUDG 15 \\
\hline PERSEUS-UDG08 & V13 & $03: 17: 44.1$ & $+41: 22: 51.1$ & 24.52 & 0.241 & 2424,2271 & 2533,2653 & WUDG $12,16,17,22$ \\
\hline PERSEUS-UDG09 & V14 & $03: 17: 45.5$ & $+41: 19: 30.2$ & 25.55 & 0.238 & 2436,2359 & 2604,2640 & WUDG $4,6,7,18,19$ \\
\hline PERSEUS-UDG10 & V15 & $03: 18: 31.1$ & $+41: 11: 31.6$ & 24.00 & 0.315 & 2424,2271 & 2533,2653 & WUDG 56,59, RUDG 16 \\
\hline
\end{tabular}

Note: In the last column, WUDG identifiers are from Wittmann et al. (2017) while the RUDG identifiers are additional UDGs from CFHT imaging (Romanowsky et al., in progress).

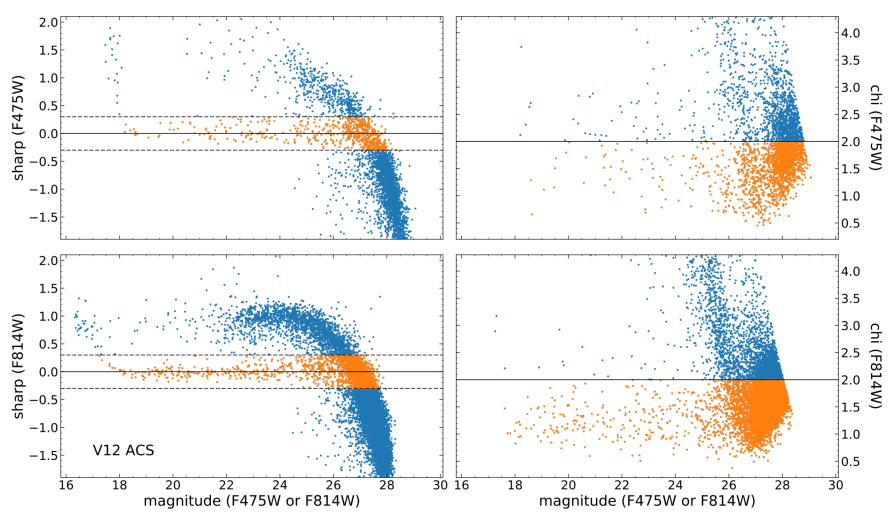

Figure 2. Illustration of the selection of GC candidates with the daophot sharp and chi indices. Magnitudes (either F475W or $\mathrm{F} 814 \mathrm{~W}$ ) are on the VEGAMAG system (see text). The example shown is for the ACS V12 field. For starlike (unresolved) objects $s h a r p$ is expected to be $\simeq 0.0$. Excluded objects categorized as nonstellar and rejected are shown with blue symbols; accepted (near-starlike) objects with small sharp, chi are in orange symbols.

stripped dwarfs, or very massive star clusters (see Mieske et al. 2012; Wittmann et al. 2016; Voggel et al. 2018, for recent discussion). At the Perseus distance, UCD candidates will be found as an automatic byproduct of our HST imaging data. From the observed scaling relation between numbers of UCDs with $M>10^{7} M_{\odot}$ and host cluster mass (e.g. Pfeffer et al. 2014, Janssens et al. 2017) Perseus should contain of order 200 UCDs. Some dozens of these are already known (Penny et al. 2011, 2012) and the results of our program are expected to yield enough UCDs in total to study the systematics of their spatial distributions and mean metallicities.

Lastly, we can expect to carry out an inventory of the M32-like compact ellipticals (cEs), a rare class of galaxy now also emerging as a topic of systematic study (Janz et al. 2016, Martinović \& Micic 2017, Zhang \& Bell 2017.

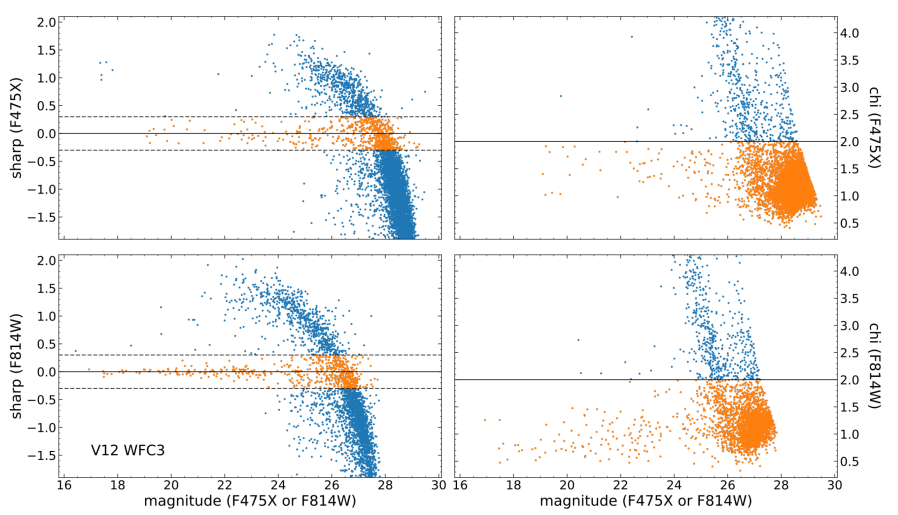

Figure 3. Selection of GC candidates with the daophot sharp and chi indices, for the WFC3 V12 field. Symbols are as in the previous figure.

Ferré-Mateu et al. 2018).

For the following discussion, we adopt a distance $d=75 \mathrm{Mpc}$ for Perseus, obtained from the redshift $5207 \mathrm{~km} \mathrm{~s}^{-1}$ corrected to the CBR frame (NED), and a Hubble constant $H_{0}=69.7 \mathrm{~km} \mathrm{~s}^{-1} \mathrm{Mpc}^{-1}$ (Hinshaw et al. 2013). From NED the mean foreground extinction is $A_{I} \simeq 0.246$, giving an apparent distance modulus $(m-M)_{I}=34.62$.

The outline of this paper is as follows: Section 2 describes the characteristics of our imaging database and the photometric measurement techniques directed towards isolating the GC candidates. Section 3 presents the color-magnitude distribution of the GCs in each target field, and photometry for a set of control fields well outside Perseus. Section 4 presents in turn the measurements of the GC effective radii, their luminosity distribution, color distribution, and radial distribution within the Perseus cluster as a whole. Section 5 concludes with a brief summary and goals for future work.

\section{DATABASE AND OBSERVATIONAL STRATEGY}


Our imaging material consists of new fields observed with HST, along with deep Subaru Hyper Suprime-Cam (HSC) imaging in gri that covers a $1^{\circ}$-diameter field, though to less depth and resolution. In the present paper, we concentrate on the HST data for the outer regions of the cluster.

Our new HST imaging data for PIPER is from $\mathrm{Cy}$ cle 25 program 15235 (PI Harris). The ACS/WFC and WFC3/UVIS cameras are used in parallel to obtain exposures of 15 pairs of fields scattered across the cluster; these pointings are shown in Figure 1 and listed in Table 1. In the Table, successive columns list the MAST Archive field identifier; the Visit number; the field coordinates (J2000) for the Primary camera pointing in each pair; their projected distance $R$ in arcminutes from NGC 1275 (the Perseus center); mean foreground extinction $A_{I}$ calculated from NED; the exposure times (in seconds) in the blue and red filters from each of ACS and WFC3; and the UDG targets included in each pointing. Exposure times in parentheses are for Visits not yet executed at time of writing. For Visits 1-5, the ACS camera is the Primary and WFC3 the Parallel; while for Visits 6-15, WFC3 is the Primary and ACS the Parallel.

The fields will be referred to below by their spacecraft Visit numbers V1-V15. The first five Visits are the ones covering the Perseus core, while the remaining 10 are the outer fields selected to cover the UDGs and, simultaneously, the Intracluster Medium. Spacecraft orientations for the outer fields were chosen to maximize the numbers of UDGs we could capture.

The core of Perseus, at center-left in the figure, is covered nearly completely out to $R \simeq 5^{\prime}(100 \mathrm{kpc}$ ) when supplemented by five additional MAST Archive ACS/WFC fields taken from program GO-10201 (PI Conselice), also shown in the figure. As can be seen in Table 1, the fieldto-field differences in foreground reddening are modest (typically $\Delta A_{I} \lesssim 0.02 \mathrm{mag}$ ) with the exception of the more heavily reddened V15.

The schedule of spacecraft visits is spread out over a two-year period from 2017 Oct to 2019 October. At time of writing, all image pairs except V2, V7, V10 have been obtained. At a scale of $22 \mathrm{kpc} / \mathrm{arcmin}$ for a Perseus distance of $75 \mathrm{Mpc}, \mathrm{V} 12$ (the most remote pointing) lies at a projected distance of $740 \mathrm{kpc}$ from Perseus center (cf. Table 1), equivalent to $40 \%$ of the Perseus virial radius (Simionescu et al. 2011).

In the present paper, we discuss the outer fields V6V15 (excluding V7, V10 not yet available) and specifically what they reveal about the population of IGCs. These images are also used to define a consistent and homogeneous set of measurement procedures for the entire set of HST data that will be discussed in later papers in our series. As will be shown below, Perseus clearly holds an IGC component, and the photometric data are of sufficient quality to characterize their color (metallicity) distribution and an initial estimate of their radial distribution within the cluster.

For most of our program goals we need to detect and characterize GCs within Perseus, either in the target galaxies or distributed throughout the diffuse ICM. Typical GCs have half-light diameters $2 r_{h} \simeq 6 \mathrm{pc}$ (Harris 1996 2010 edition), which at $d=75 \mathrm{Mpc}$ translates to $0.014^{\prime \prime}$, almost an order of magnitude smaller than the natural $0.1^{\prime \prime}$ resolution of HST. This means that most GCs belonging to the Perseus galaxies will be nearstarlike (that is, unresolved) objects, which is a major advantage for carrying out photometry and for isolating them from the field contaminants that are dominated by faint, small background galaxies. In addition, GCs fall in a relatively narrow range of color index, permitting further rejection of very blue or very red contaminants. This combination of object morphology and color gives us a very effective filtering procedure to isolate the GC population with a low level of residual contamination (that is, a level where the contaminating non-GC population makes only a second-order contribution to the total, as will be seen in Section 3 below).

In all cases our program employs exposures in filters that maximize signal-to-noise and can, if desired, be transformed to standard $(B, I)$ for easy comparison with previous GC data in the literature. The adopted filters are (F475W, F814W) for ACS/WFC; and (F475X, F814W) for WFC3/UVIS. Though WFC3 F475X has rarely been used, it is significantly broader than $\mathrm{F} 475 \mathrm{~W}$ and still transforms well to standard $B$ (Harris 2018). For each field, either three or four dithered sub-exposures are taken in each filter to give a final image effectively free of cosmic rays and other artifacts. In what follows, we will refer to the exposure pairs as the $B$ and $I$ images, although we present the results in the natural filter magnitudes.

The procedure for detection and photometry of the GC candidates is similar to the steps outlined in, e.g., Harris (2009); Harris et al. (2016) or HM17. We start with the CTE-corrected, drizzled and combined *.drc images provided by MAST. First, the individual $B$ and $I$ exposures are registered and combined to create a master image in each filter. In the pyraf implementation of daophot, daofind is used independently on each filter to generate findlists of objects. Then follows the normal sequence of phot (through a $r=2 \mathrm{px}$ aperture), psf, and allstar. The point-spread function (PSF) was defined empirically on each $B$ and $I$ image individually from a combination of typically 30-50 moderately bright, uncrowded stars. Tests were made to compare results adopting either a uniform PSF or one depending on position $(x, y)$ in the image, but with entirely similar results; the uniform-PSF mode was adopted. The allstar magnitudes were then corrected to equivalent large-aperture magnitudes. The appropriate photometric zeropoints for each filter and for the exact dates of the exposures, as taken from the STScI webpage zeropoint calculators, were then added to put the data onto the VEGAMAG system and in the natural filter magnitudes (F475W, F814W for ACS, and F475X, F814W for WFC3).

Final rejection of distinguishably nonstellar objects was done with the sharp, chi, and err parameters generated by allstar, examples of which are shown in Figures 2 and 3. Sharp is defined such that starlike objects (ones closely matching the PSF) will appear near $\simeq 0$, and exclusion regions can then be defined to reject much smaller or more extended sources as shown in the Figure. Any objects with $\mid$ sharp $\mid>0.3$, chi $>2$, or err $>0.3$ on either filter were rejected 1$]$ Extreme care was taken

\footnotetext{
${ }^{1}$ In practice, these three parameters are correlated. That is, any object rejected because of high sharp would also often have been rejected for high chi, err. In general, however, the sharp culling was
} 
through the iterative PSF definition step to ensure that the brighter, uncrowded stars in the image gave sharp values very close to zero. The internal scatter of the objects in the desired 'starlike' sequence at sharp $=0$ differs slightly from one field and filter to another, so fixed exclusion boundaries at sharp $= \pm 0.3$ were chosen. Setting the boundaries there ensured that we included virtually all objects in the starlike sequence in every image, while also excluding the same numbers of contaminating field objects (see below).

Finally, the culled object lists in each filter were matched to within $\Delta r=2 \mathrm{px}$ in position, further rejecting any objects not appearing in both filters. The entire combination of steps described above is effective not just at culling clearly nonstellar objects, but also at removing spurious detections near the CCD chip edges or within the bright cores of any Perseus member galaxies falling within the fields.

Saturation of starlike objects occurs for magnitudes F814W $\lesssim 18$, as can be seen in Fig. 2. However, as will be seen below, the brightest GCs that are our main targets lie safely fainter than this, at $\mathrm{F} 814 \mathrm{~W} \gtrsim 22$.

Photometric completeness of detection, and thus the effective limiting magnitudes of our data, was evaluated by adding populations of artificial stars (scaled PSFs) into the images through daophot/addstar. The completeness ratio $f(m)$ is the number of detected artificial stars at a given magnitude $m$ divided by the number of stars inserted at $m$. All of these target fields are very sparsely populated and the sky intensity level is low and quite uniform, as we illustrate in Figure 4. Although many small, faint background galaxies can be seen scattered across the frame, the numbers of similarly faint unresolved objects are quite small, and crowding is not an issue for any part of the data. In addition, all the fields have nearly identical exposure times. Under these almost ideal circumstances, the completeness fraction will drop quite steeply from near-100\% to near-zero over a short magnitude range. We model $f(m)$ with the simple two-parameter function (Harris et al. 2016)

$$
f=\left(1+\exp \left(\alpha\left(m-m_{0}\right)\right)\right)^{-1}
$$

where $m_{0}$ is the magnitude at which $50 \%$ of the objects are detected and $\alpha$ measures the steepness of falloff of the completeness curve. Examples of the completeness test results are shown in Figure 5 , while the best-fit parameters and their uncertainties are listed in Table 2, For the ACS data we find $m_{0} \simeq 28.0(F 475 W), 27.0(F 814 W)$, while for WFC3 (the more blue-sensitive of the two cameras) $m_{0} \simeq 28.4(F 475 X), 26.7(F 814 W)$.

\section{DETECTING THE IGCS}

Any GCs found in our $(8+8)$ target fields are remote from the major Perseus galaxies and therefore will be associated either with dwarf galaxies or the even less luminous UDGs in those fields, or with the IntraCluster Medium. Decades of previous work on GC populations in other galaxies have established a remarkably consistent set of properties of GC systems, including their luminosity distributions, color (metallicity) distributions, and GC scale sizes. We look briefly at each of these

the first and most stringent step. By comparison, further culling by $c h i$, err rejected only an additional few percent of the objects. properties in turn, and in doing so build up the case that the Perseus IGC population is clearly detected.

From analysis of a set of HST Archival fields (some of which are marked in Fig. 1), HM17 found suggestive evidence for the presence of 1GCs. However, as noted above, their preliminary findings relied in the end on a few WFPC2 fields with much shallower exposures than the ones in our program, and on ACS fields located in the core region near the giants NGC 1272 and 1275, making it difficult to isolate a significant sample free of contamination from Perseus galaxies. The data in our program are better designed to find the intergalactic component, however much of it there is.

\subsection{Color-Magnitude Data}

After the image matching and rejection of nonstellar objects as described above, the resulting color-magnitude diagrams (CMDs) for our eight pairs of fields are shown in Figure 6. Here, the data from both cameras have been put onto a common photometric system, which we have adopted as $(\mathrm{F} 475 \mathrm{~W}, \mathrm{~F} 814 \mathrm{~W})_{A C S}$. As noted above, the red filter for the WFC3 images was F814W, whose effective wavelength is very similar to the equivalent filter for $\mathrm{ACS}$ and thus $\mathrm{F} 814 \mathrm{~W}(\mathrm{WFC} 3) \simeq \mathrm{F} 814 \mathrm{~W}$ (ACS), which in turn are closely equivalent to the Johnson/Cousins I band (Harris 2018). The blue filter for the WFC3 images was F475X, chosen as noted above for its wider bandpass. Though it maximizes the blue limiting magnitude for a given exposure time, it is significantly redder than F475W and thus needs transformation into F475W $\mathrm{W}_{A C S}$. From the equations in Sirianni et al. (2005) and Harris (2018), we obtain

$$
F 475 W_{A C S}=F 475 X_{W F C 3}+0.16(F 475 X-F 814 W)
$$

which has been applied to all the WFC3 measurements.

In each field of Fig. 6 a distinct population of objects can be seen at intermediate color (F475W-F814W) $\simeq 1.8$ / and magnitude range $\mathrm{F} 814 \mathrm{~W} \gtrsim 23$, near the expected values for GCs. A composite CMD including data from all 16 individual fields is shown in Figure 7. where now each field has been individually dereddened so that the data can be strictly matched together. The CMD in Fig. 7 does, however, exclude a few small concentrations of points around identifiable small galaxies in the target fields. These exclusion regions are shown in Figure 8. The distribution of these GC candidates across the CMD is quite insensitive to the exact placement of the spatial boundaries in Fig. 8 around the dwarf galaxies, because of the well known property of GCs in dwarfs to be almost entirely low-metallicity ones (Peng et al. 2011). The combined CMD represents a total area of $150 \operatorname{arcmin}^{2}$.

In this combined diagram, the GC candidate sequence now stands out more clearly. The large swath of points crossing the upper right of the CMD is dominated by foreground-star contamination (see below), but fortunately this region is well separated from the target GCs. Taking into account the rapidly increasing scatter in color for $I>26$ and the completeness limits in each filter, we isolate a "GC candidate" region generously defined by the box $22.0<F 814 W_{0}<25.5$, $1.0<(F 475 W-F 814 W)_{0}<2.4$. Within this box the photometry is also highly complete $(>90 \%)$. The 


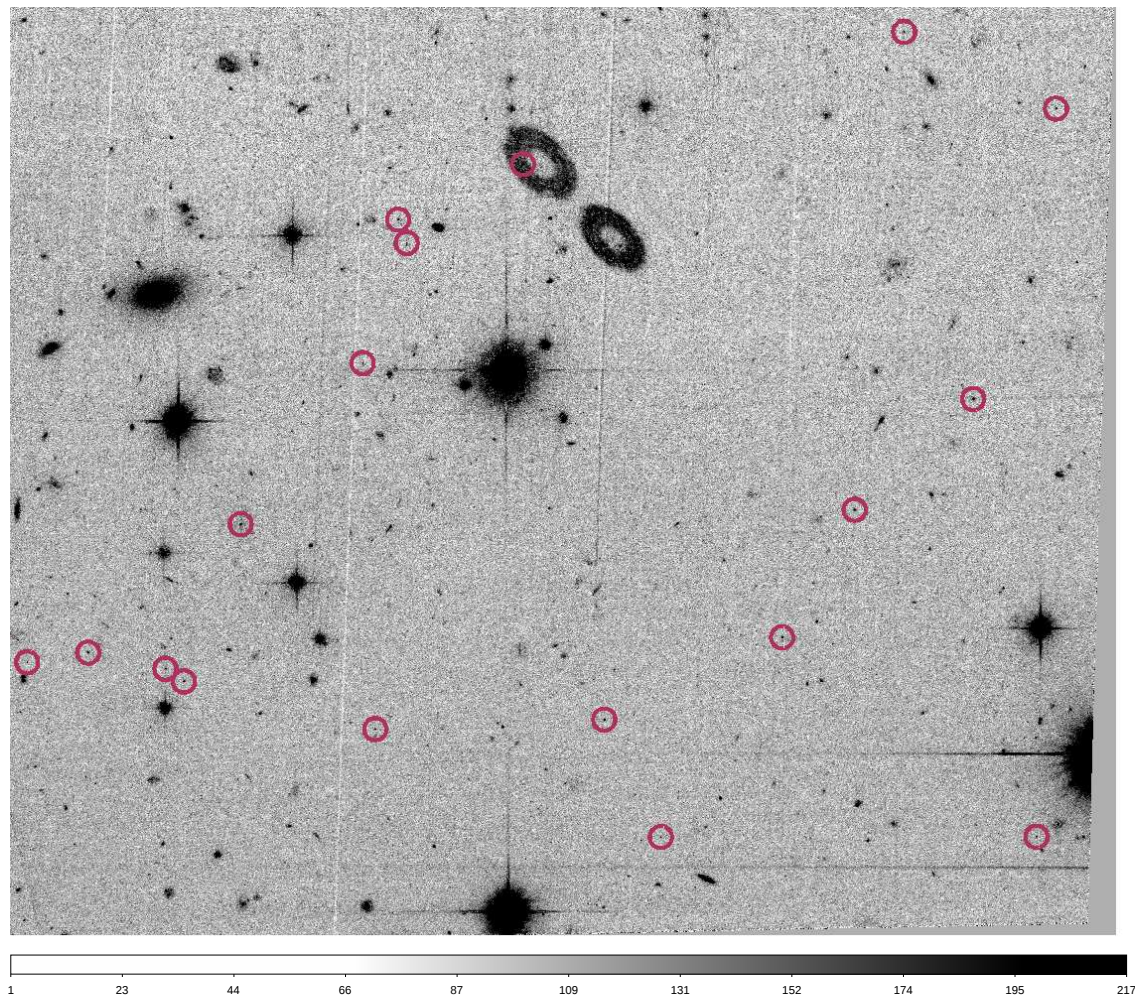

Figure 4. A section of the V15 field from the ACS camera, in F814W. The segment shown is $100^{\prime \prime}$ across. The faint objects marked with the colored circles are GC candidates, which are starlike objects with $I<26$ and colors in the range $1.0<(F 475 W-F 814 W)_{0}<2.4$. The two oval rings above center are ghost images of adjacent bright stars.

boundaries of this region correspond to absolute magnitudes and intrinsic colors $-12.4<M_{I}<-8.9$ and (transformed color range) $1.2<(B-I)_{0}<2.9$. For comparison, the GCs in a sample of Brightest Cluster Galaxies (Harris 2009) populate the range $M_{I}>-12$ and $1.4 \lesssim(B-T)_{0} \lesssim 2.3$, further indicating that we are seeing a population of GCs. As will be seen below, the candidate GCs almost all fall in a somewhat narrower color range $1.0<(F 475 W-F 814 W)_{0}<2.0$. However, in anticipation of later papers in this series, which will concentrate on the GC populations around the Perseus giant ETGs that have much larger numbers of more metal-rich GCs that may extend redder, we keep a slightly more generous color boundary at the red end.

\subsection{Background Contamination}

The fraction of this GC candidate sample that consists of actual Perseus GCs depends on the residual level of field contamination in the photometry. This contamination consists of foreground stars and very faint, small background galaxies that managed to pass through the culling steps described above. Ideally we would like to assess the contamination level from background control 


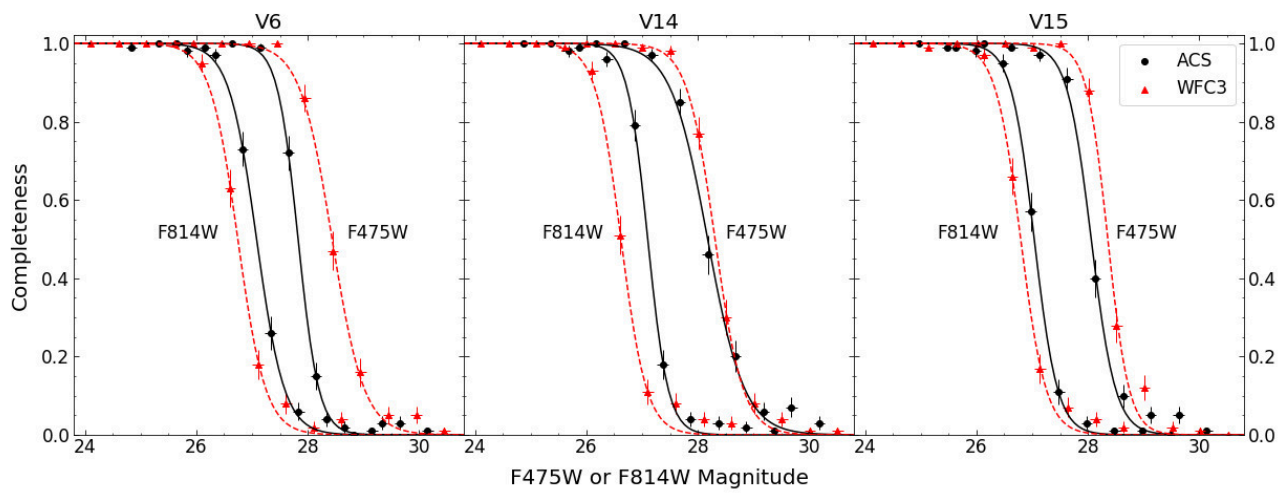

Figure 5. Photometric completeness tests for the ACS and WFC3 data. The completeness $(f)$ is the fraction of starlike objects at a given $B$ or $I$ magnitude that were detected, and is plotted versus magnitude. Black points and curves show the results for ACS, and red triangles and curves show the results for WFC3. The completeness drops steeply from $100 \%$ to near-zero over an interval of roughly a magnitude.

Table 2

Photometric Completeness

\begin{tabular}{lll}
\hline Field & $\alpha$ & $m_{0}$ \\
& & \\
\hline & & \\
V6 ACS F475W & $5.4(0.2)$ & $27.83(0.01)$ \\
V6 ACS F814W & $4.0(0.2)$ & $27.08(0.02)$ \\
V6 WFC3 F475X & $3.5(0.2)$ & $28.44(0.02)$ \\
V6 WFC3 F814W & $4.0(0.3)$ & $26.74(0.02)$ \\
V14 ACS F475W & $3.0(0.3)$ & $28.18(0.03)$ \\
V14 ACS F814W & $5.5(0.3)$ & $27.10(0.01)$ \\
V14 WFC3 F475X & $4.0(0.2)$ & $28.31(0.01)$ \\
V14 WFC3 F814W & $4.4(0.5)$ & $26.62(0.02)$ \\
V15 ACS F475W & $4.6(0.5)$ & $28.06(0.02)$ \\
V15 ACS F814W & $4.8(0.2)$ & $27.04(0.01)$ \\
V15 WFC3 F475X & $5.3(0.6)$ & $28.36(0.03)$ \\
V15 WFC3 F814W & $4.4(0.6)$ & $26.79(0.02)$
\end{tabular}

Table 3

CONTROL Fields

\begin{tabular}{lllllllll}
\hline Field & \multirow{2}{*}{ Identifier } & \multirow{2}{*}{ RA } & \multicolumn{2}{l}{ Dec } & $\ell$ & $b$ & \multirow{2}{*}{$t_{I}(\mathrm{sec})$} & $t_{I}(\mathrm{sec})$ \\
\hline HFF2 & MACSJ0416.1-2403 & $04: 16: 33.1$ & $-24: 06: 48.7$ & 327.3 & +34.3 & 0.134 & 3858 & 3855 \\
HFF3 & MACSJ0717.5+3745 & $07: 17: 17.0$ & $+37: 49: 47.3$ & 180.5 & +21.6 & 0.104 & 3858 & 3855 \\
HFF4 & MACSJ1149.5+2223 & $11: 49: 40.5$ & $+22: 18: 02.3$ & 230.5 & +75.6 & 0.039 & 3855 & 3855 \\
HFF5 & Abell S1063 & $22: 49: 17.7$ & $-44: 32: 43.8$ & 349.4 & -60.6 & 0.021 & 3855 & 3855 \\
HFF6 & Abell 370 & $02: 40: 13.4$ & $-01: 37: 32.8$ & 173.7 & -52.9 & 0.055 & 3858 & 3855 \\
SS22A-PAR & GO11636 & $22: 17: 14.5$ & $+00: 14: 12.3$ & 63.8 & -44.3 & 0.103 & 3000 & 3000 \\
& & & & & & & & \\
\hline
\end{tabular}

fields near but outside Perseus, taken with ACS or WFC3 and with $B, I$ filters and similar exposure times to our program fields. However, we have found no such ideal material in the MAST archive. Instead, as a preliminary measure we use data for six different fields listed in Table 3 that do have the right combinations of filters and exposure times, but which lie in widely different parts of the sky. These include five Hubble Frontier Fields (Lotz et al. 2017) and one from program GO-11636 ${ }^{2}$ All of these are Parallel exposures with the ACS/WFC camera, and in all six cases they fall on 'blank' fields free of any major galaxies or clusters of galaxies. We have subselected individual exposures from each program adding up to total exposures similar to but a bit longer than

2 One other HFF field, targeted on Abell 2744, is not suitable for our purposes because its Parallel image is heavily contaminated by galaxies in this very extensive cluster, and its own IGCs. 

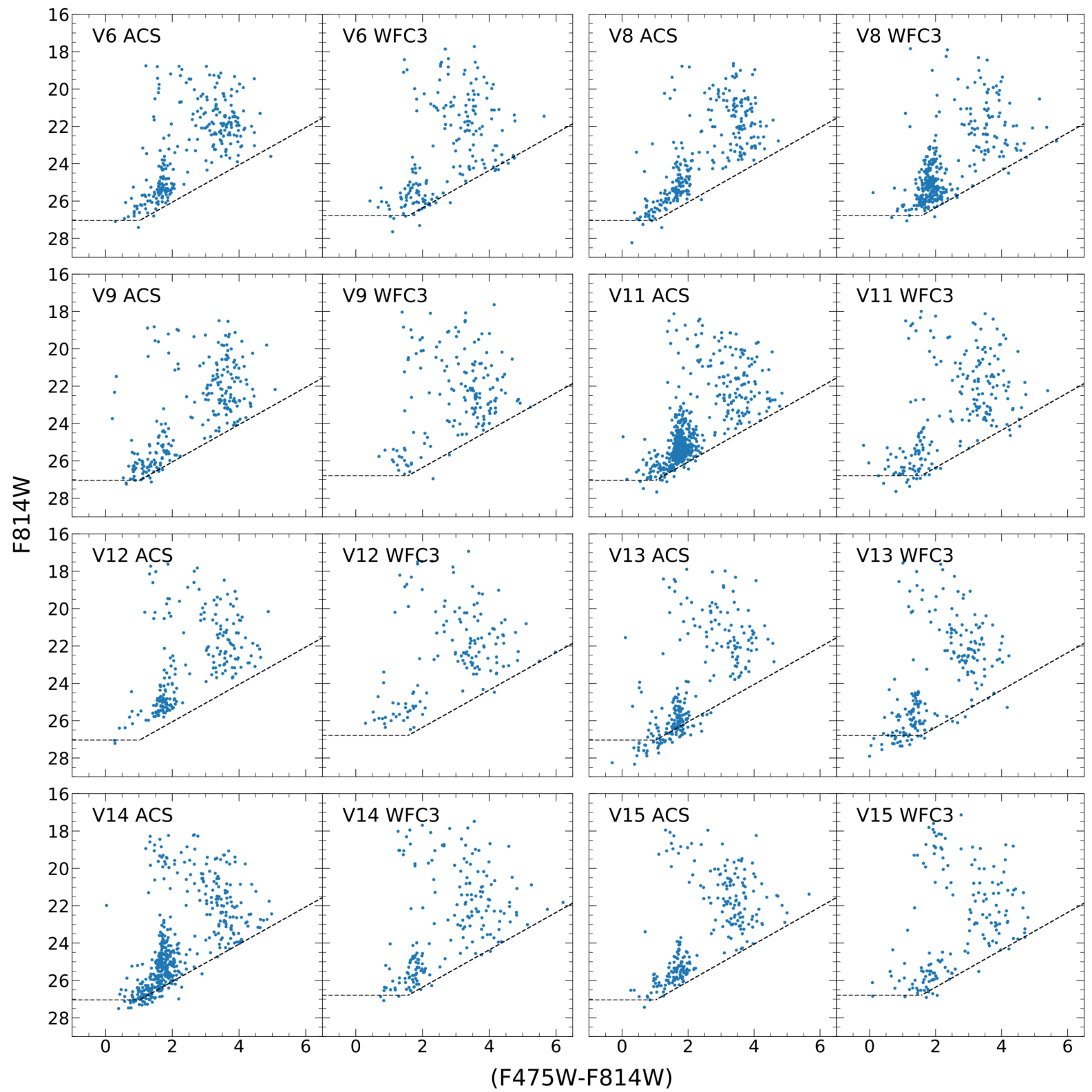

Figure 6. Color-magnitude data for starlike (unresolved) objects in eight individual pairs of fields. The data are not de-reddened. The dashed line indicates the average $50 \%$ detection completeness level for the ACS fields as a rough guide to the limits of our data.

our Perseus target fields, so that they will have limiting magnitudes slightly deeper than our program fields. In Table 3, we list the field coordinates, exposure times in the blue and red filters, and foreground extinctions $A_{I}$.

We have generated and measured the images for all of these control fields with exactly the same procedures as for our Perseus targets, including the same process of object selection and culling of nonstellar objects. The images for the five HFF fields are taken in $(\mathrm{F} 435 \mathrm{~W}$, $\mathrm{F} 814 \mathrm{~W})$, and the images for SS22A-PAR are taken in (F475W, F814W). The HFF data were transformed to the $(\mathrm{F} 475 \mathrm{~W}, \mathrm{~F} 814 \mathrm{~W})$ system through

$$
F 475 W=F 435 W-0.174(F 435 W-F 814 W)
$$

again derived from the equations from Sirianni et al. (2005). In Figure 9, the results for the six control fields are shown individually, while in Figure 10, all six are individually dereddened and combined. This combined CMD represents a total area of $70 \operatorname{arcmin}^{2}$.

In total, 93 objects fall clearly within the GC-candidate region defined above, making an average of 15.5 per ACS field or equivalently 10 per WFC3 field. All of the six fields, however, are at higher Galactic latitude than is Perseus, and in principle the numbers of contaminating objects falling within the GC candidate selection box could be a function of latitude if they are dominated by Galactic foreground stars rather than faint background galaxies. An empirical test is shown in Figure 11, where the numbers per field are plotted versus the absolute value of Galactic latitude. Notably, the numbers do not depend significantly on $b$. indicating that the contaminants within our magnitude and color range of interest 


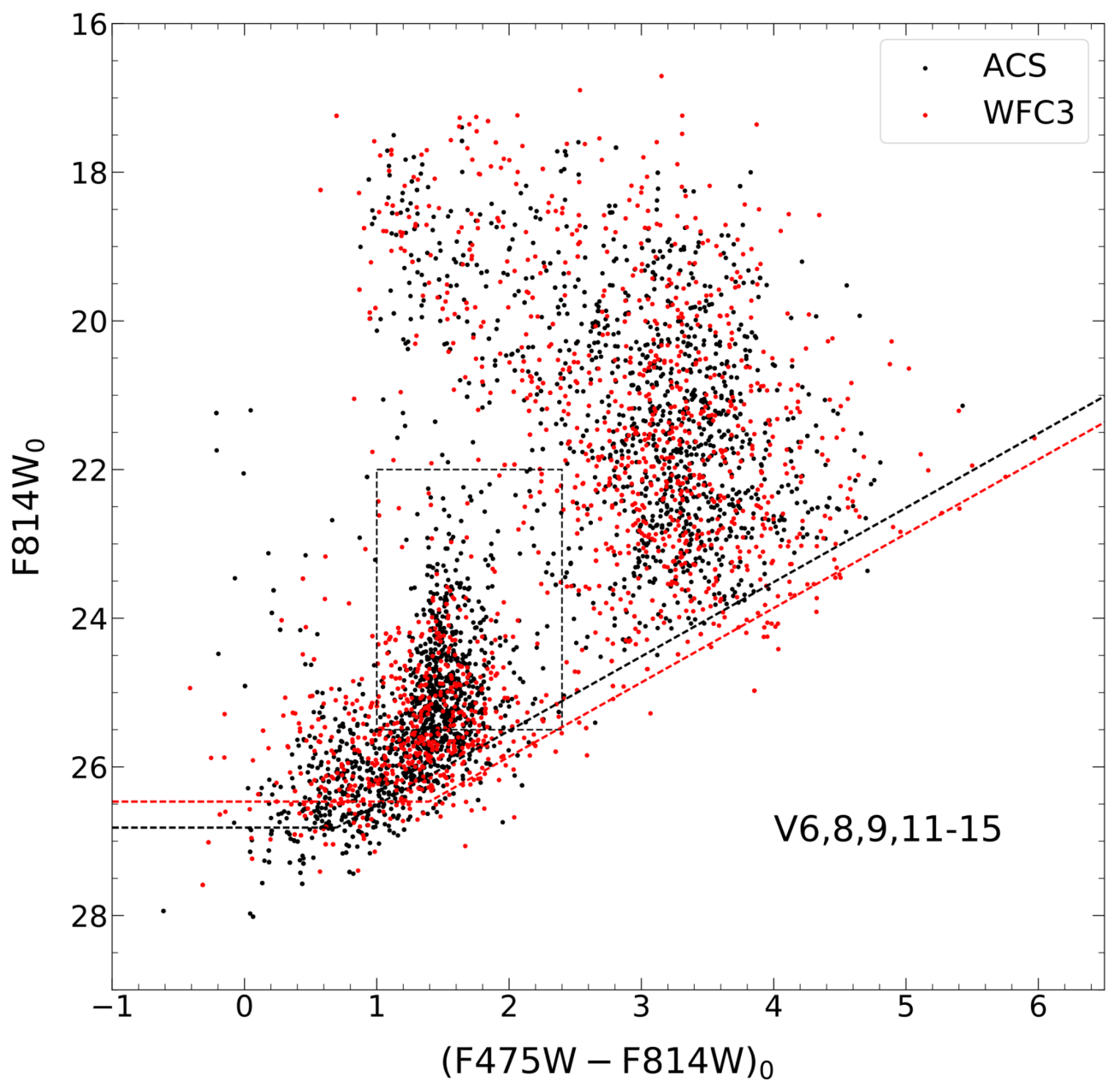

Figure 7. Composite CMD for all eight visits discussed in the present paper. Black points are the ACS images combined, red points are the WFC3 images. Objects falling within any of the circles shown in the next Figure are excluded to remove any GCs belonging to the dwarf galaxies in the fields. The average $50 \%$ completeness thresholds for each camera, established from the completeness tests described in the text, are shown as the dashed lines (black for ACS, red for WFC3). The box outlined in the black thin-dashed line marks the GC candidate region defined in the text.

are primarily from the Galactic halo rather than the disk. Over the entire $150 \operatorname{arcmin}^{2}$ area of our Perseus fields, these numbers would therefore suggest that we should expect $\simeq 200$ contaminants in total within the candidate box. Averaging the 6 fields gives a mean number density $\sigma_{b k g d}=(1.33 \pm 0.21) \operatorname{arcmin}^{-2}$. The uncertainty of this mean value is about 1.5 times higher than expected from simple Poisson statistics, which we take to be due to intrinsic cosmic scatter. Substructure in the Milky Way halo, and clustering of faint background galaxies over scales of a few arcminutes, will both contribute to this increased variance.

As an additional check on this estimate, we use the TRILEGAL population model of the Milky Way (Girardi et al. 2005) to gauge the numbers of foreground stars at the latitude and longitude of Perseus. These results are also shown in Figure 10 for a projected area on the sky of $150 \mathrm{arcmin}^{2}$, equal to the total area of our $(8+8)$ Perseus fields. In this model, 143 stars fall within the GC candi- date box, which makes up roughly $70 \%$ of the total from the control fields given above. At face value, the TRILEGAL counts therefore suggest that only $\simeq 30 \%$ of the control-field counts are from faint background galaxies small enough to pass through our selection filters.

Perhaps more importantly, the distribution of model stars in Fig. 10 is useful for showing where the CMD is most affected by contamination, which is the large swath of points at upper right. These are largely faint lower main sequence stars at various distances in the Milky Way disk (and to a lesser extent the halo), and their total numbers are highly sensitive to Galactic latitude. Fortunately, the Perseus GC candidates lie bluer and fainter than these stars, in a region of the CMD where very little contamination is present.

Within the GC candidate box, there are a total of 824 objects after rejection of ones very near the obvious dwarf galaxies, as noted above. In the same box, the data from the control fields predict that $\sim 200$ of these 

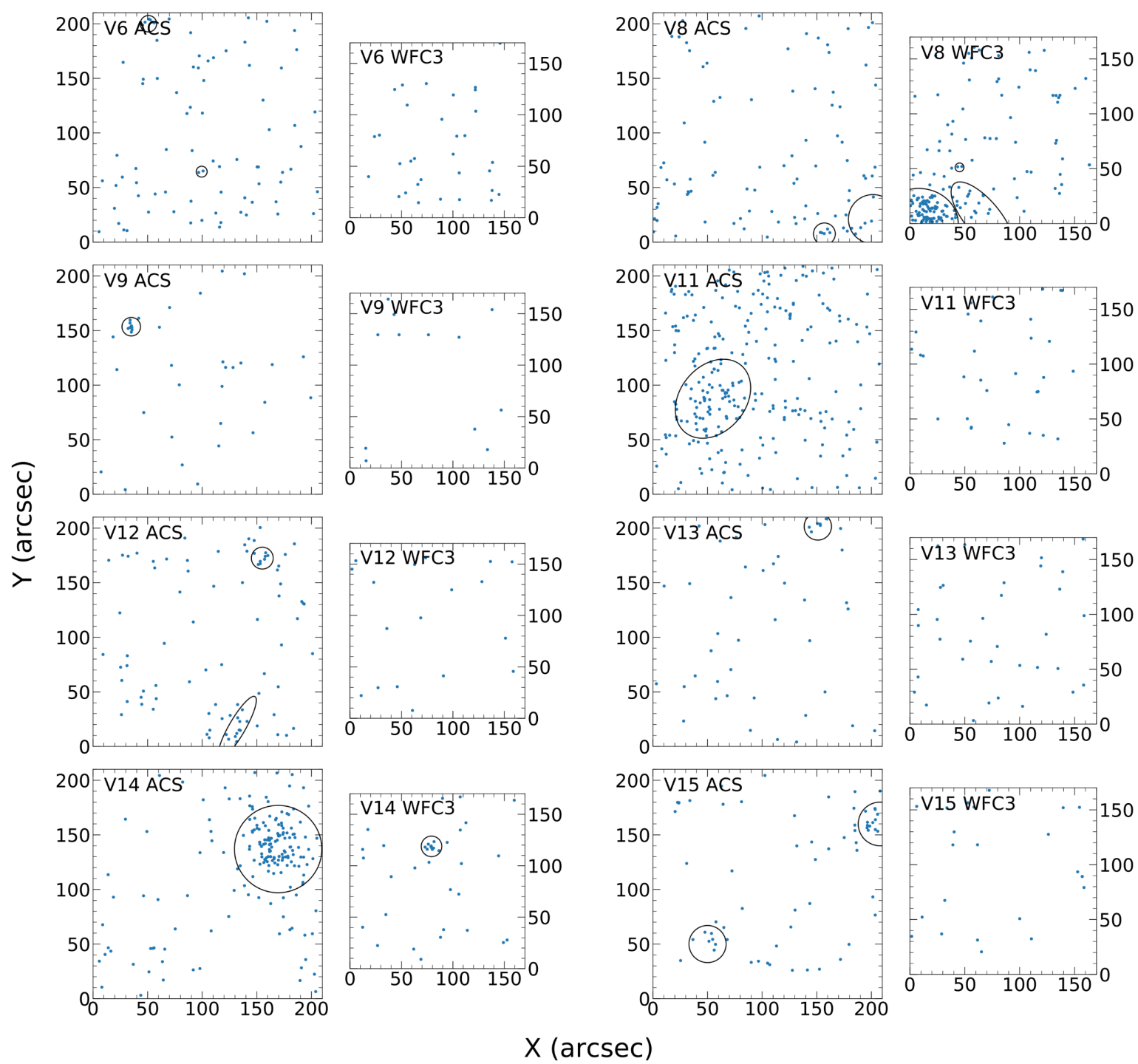

$X(\operatorname{arcsec})$

Figure 8. Locations of objects in each field that fall within the color and magnitude range for normal globular clusters $(1.0<(F 475 W-$ $\left.F 814 W)_{0}<2.0,22.5<F 814 W<25.5\right)$. For each pair of panels, the left shows the ACS camera, and the right shows the WFC3 camera. Black circles and ellipses indicate the boundaries of small galaxies that have detectable GC populations of their own; objects inside these regions are excluded from the composite CMD shown in Fig. 7

should be "background", for a contamination rate of $24 \%$. However, the background objects are quite evenly distributed in color across the box (Fig. 10) whereas the great majority of the GC candidates are in the color range $1.0<(F 475 W-F 814 W)_{0}<2.0$. In this narrower color range, there are 775 candidates but an estimated 156 background objects in the same $150 \mathrm{arcmin}^{2}$ area, for a somewhat lower contamination fraction of $20 \%$.

These results indicate that the contamination level in our sample of outer fields is small though not negligible. It is clear, however, that sparsely spread as they are, IGCs are present and clearly measurable in these outlying Perseus fields.

\section{RESULTS: CHARACTERIZING THE IGCS}

The HST data give high enough resolution, depth, and precision to permit measurement of several characteristics of the IGCs and to make a series of tests of how consistent their properties are compared with those in galaxies. These include their scale sizes (half-light radii); their distribution in luminosity (the GCLF); and their metallicity distribution as represented by color indices. All of these results are consistent with the conclusion that the IGCs are a generally normal population of GCs.

\subsection{Effective Radii}

Although by definition all the objects in the GC candidate list are reasonably well matched to the PSF shape within the specified range $\mid$ sharp $\mid<0.3$ as discussed above, estimates of intrinsic radii can be obtained by a more detailed fit of the PSF to each object. We employ 

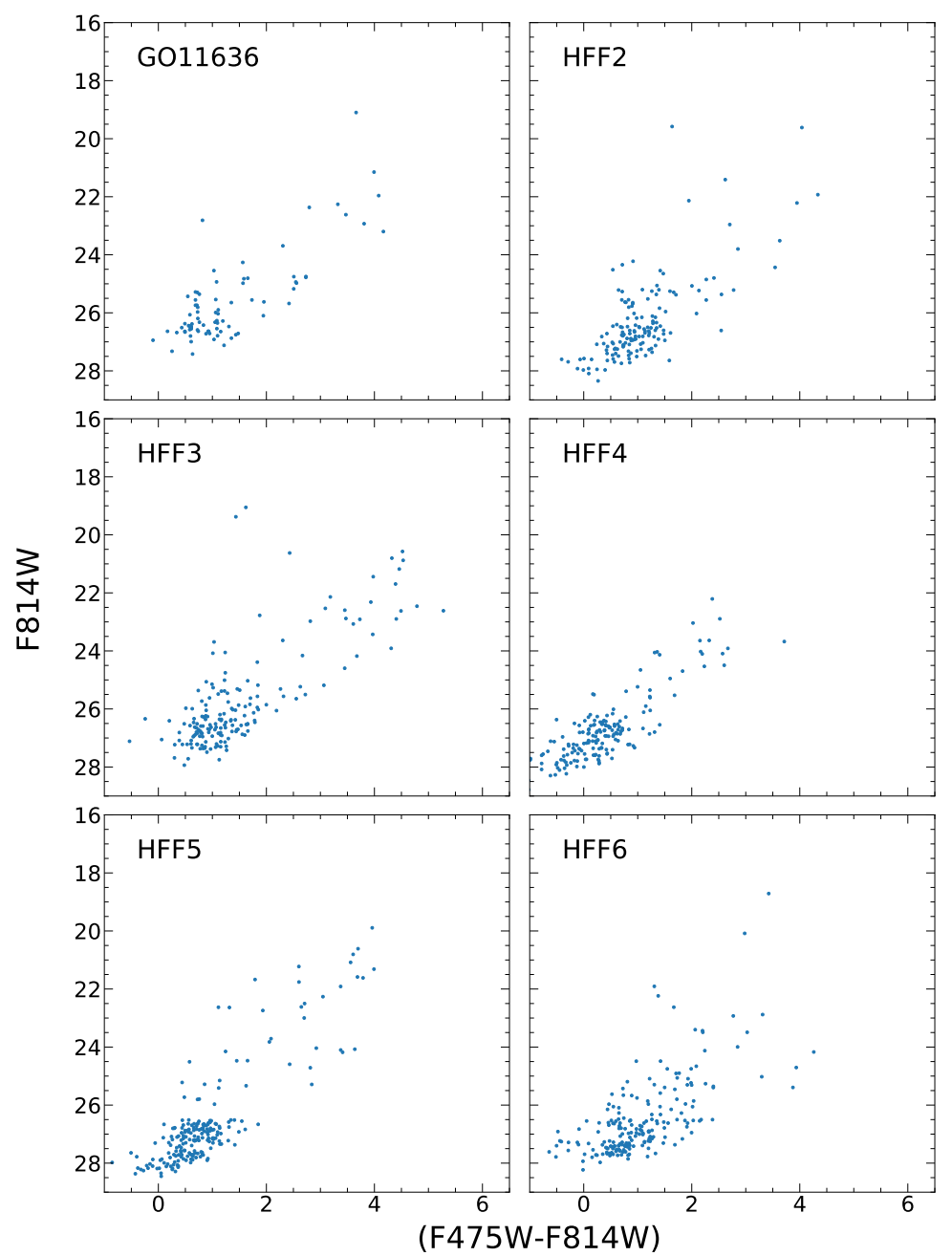

Figure 9. CMDs for the six HST/ACS control fields listed in Table 3 Photometric measurement and object selection were carried out with exactly the same procedures as for the Perseus target fields. Compare with Fig. 6

the code ishape (Larsen 1999) for this purpose, following many previous instances of its use in the literature for GCs in remote galaxies (e.g. Larsen 1999: Larsen et al. 2001, Georgiev et al. 2008, Harris 2009, Lim et al. 2013, among others).

To run ishape we assume an intrinsic GC profile with central concentration $c=r_{t} / r_{c}=30$ or $\log c=1.5$ (KING30 in the code notation), which is an average value for GCs (Harris 1996, 2010 edition). This assumed profile is then convolved with the individual PSF for each field and matched to each object, varying the assumed fwhm until a best fit is achieved. We run ishape only on the $I$-band images because of their higher SNR.

The HST resolution of fwhm $=0.1$ " is equivalent to 36 pc in linear scale. Conservatively, ishape can measureably resolve a near-starlike object if its fwhm is larger than about $10 \%$ of the stellar fwhm (see Larsen 1999 Harris 2009). For a KING30 model profile, the halflight radius is $r_{h}=1.48 \mathrm{fwhm}$. At the Perseus distance $d=75 \mathrm{Mpc}$, this resolvable limit then corresponds to $r_{h}(\mathrm{lim}) \simeq 5$ pc. In the Milky Way, the GC $r_{h}$ distribution peaks near $3 \mathrm{pc}$ and almost none are smaller than $r_{h}=1 \mathrm{pc}$, but the distribution has a long tail to larger radii extending beyond $10 \mathrm{pc}$. Thus we should expect that many GCs in our candidate list will remain starlike, but also that the larger GCs will be measurably resolved. As a direct consistency test, ishape was also applied to the artificial stars used in the completeness tests described above. In principle, for these the fitted fwhm estimates should all be zero with some stochastically generated scatter.

In Figure 12 the $r_{h}$ measurements for all fields are displayed in histogram form. The distribution of Perseus IGCs is clearly more extended than the histogram for the artificial stars, which by definition are completely unresolved; $97 \%$ of these stars fall within the first bin $\left(r_{h}<1\right.$ pc). Many of the GC candidates also fall within $r_{h}<1$ pc: these should be a combination of genuine stars from field contamination (see discussion above), plus genuine but small GCs.

Notably, in the 'resolved' range $r_{h}>5 \mathrm{pc}$, the shape of the Perseus histogram resembles the high $-r_{h}$ tail of the Milky Way distribution also shown in Fig. 12. Although the scale sizes of GCs occupy a large range from $\lesssim 1 \mathrm{pc}$ up to $10 \mathrm{pc}$ and more, their average size increases with galactocentric distance (Gómez \& Woodley 2007; Harris 2009 Harris et al. 2010), due to weaker galactic tidalfield limits at larger distance. When this trend is coupled 


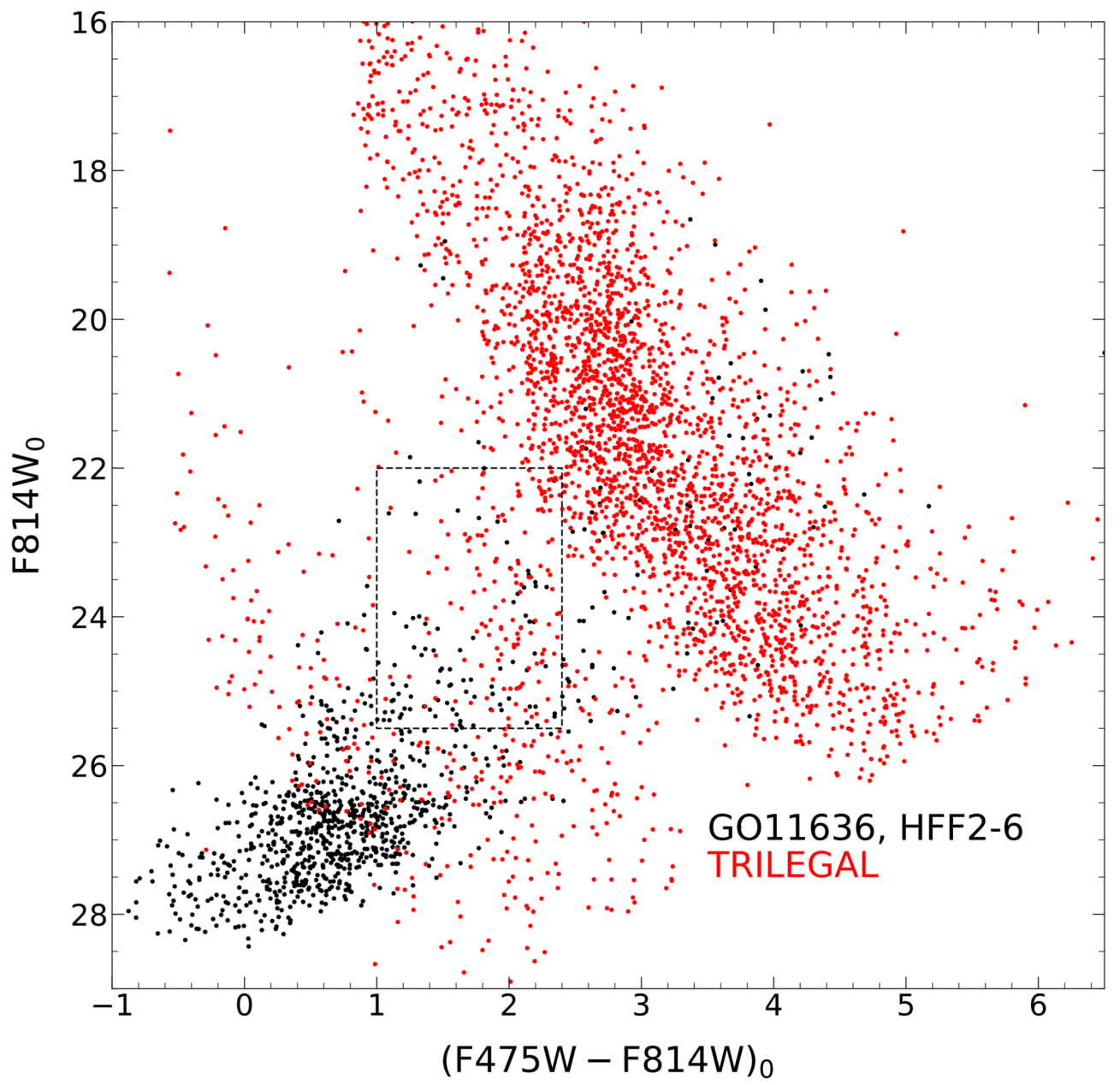

Figure 10. Combined CMD for expected field contamination. Black dots are from the six control fields listed in Table 3 while red dots are a sample of the predicted population of Milky Way foreground stars from the TRILEGAL model and described in the text. The box marked out by the dashed line indicates the GC-candidate region for Perseus; compare with Figure 7.

with the prediction that IGCs are clusters stripped away from galactic halos during interactions, IGCs should then be expected to have larger scale sizes than the bulk of the GC population in the inner regions of galaxies. In the Milky Way, for example, the median size for 95 GCs lying within $R_{g c}<8 \mathrm{kpc}$ is $r_{h}($ med $)=2.5 \mathrm{pc}$, while for 59 clusters with $R_{g c}>8 \mathrm{kpc}, r_{h}($ med $)=4.7 \mathrm{pc}$, almost twice as large as for the inner halo. To the extent allowed by the resolution limits of our imaging, we conclude that the scale-size distribution for the Perseus candidates is quite consistent with their identification as GCs.

Intercomparisons of scale sizes $\left(r_{h}\right)$ of GCs within other galaxies have been carried out for numerous galaxies in Virgo, Fornax, and other relatively nearby environments (Jordán et al. 2005; Masters et al. 2010, Harris 2009, Gómez \& Woodley 2007). 'These studies show close consistency of the $r_{h}$ distributions between galaxies, including the Milky Way itself (enough so that the $r_{h}$ distribution can be used as a rough distance indicator; see Jordán et al. 2005). Instead, we would like in princi- ple to compare the distribution in Fig. 12 with other IGC populations. The only such high-quality data available are the scale size measurements for just four IGCs in Virgo measured from HST imaging (Williams et al. 2007) (though see also Ko et al. (2017) for two other IGCs measured from ground-based imaging): these four have $r_{h}=2.0,3.4,8.0$, and $9.6 \mathrm{pc}$. All of these fit comfortably within the Perseus IGC range, and their average $\left\langle r_{h}\right\rangle=5.8 \mathrm{pc}$ is near the peak of the Perseus IGC distribution.

\subsection{Luminosity Function}

Old GC populations consistently follow a Gaussian-like luminosity function (LF) in number per unit magnitude (e.g. Jordán et al. 2007; Villegas et al. 2010; Harris et al. 2014 ), so an additional test of the nature of the Perseus candidates is the shape of their LF. This is shown in Figure 13 for the combined candidate sample. At present, only a rough test can be made since the photometric limit falls short of the normal GCLF peak frequency (turnover 


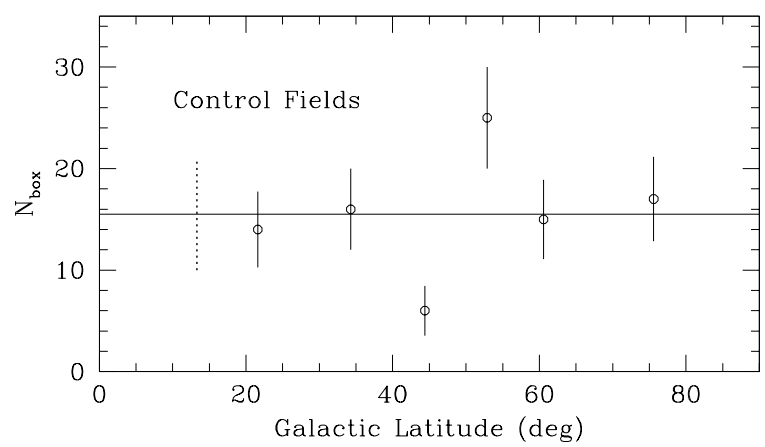

Figure 11. Number of measured objects falling within the GC candidate box, in each of the six control fields, plotted versus absolute Galactic latitude $|b|$. Errorbars assume $N^{1 / 2}$ Poisson statistics. The horizontal line at $N_{b o x}=15.5$ indicates the mean value over the six fields. The latitude of the Perseus cluster $\left(b=-13.3^{\circ}\right)$ is marked with the vertical dotted line at left.

point). The previous work of HM17 shows similar results, though their data are mostly from the Perseus core galaxies.

The turnover (peak) absolute magnitude of the GCLF, and its dispersion $\sigma$ have typical values $M_{I}^{0} \simeq-8.4$ and $\sigma \simeq 1.2$ for galaxies comparable to the Milky Way. However, both the turnover and dispersion depend systematically on host galaxy luminosity (cf. Villegas et al. 2010) such that larger galaxies have progressively more luminous turnovers (at the rate of $\simeq 0.04 \mathrm{mag}$ per galaxy absolute magnitude) and broader GCLFs (at the rate of $\simeq 0.1$ mag per galaxy absolute magnitude). Recent modelling indicates that the biggest contributions to the ICL are stars stripped from dwarf-sized to intermediate-sized galaxies (Harris et al. 2017a; Ramos-Almendares et al. 2018). Such galaxies are $3 \mathrm{mag}$ or more fainter than the giants that much of the previous GCLF literature has concentrated on. Thus in principle, accurate determination of the GCLF parameters for the IGC component would provide an additional observational test of its origin. However, if the photometric limit falls short of the GCLF turnover as is the case here, both $I^{0}$ and $\sigma$ cannot be reliably solved simultaneously (e.g. Hanes \& Whittaker 1987), and for the present we are limited to a simple consistency test. In Fig. 13 an illustrative curve is shown for a Milky-Way-like system with turnover $I^{0}=26.2$ and dispersion $\sigma=1.2$. Again, the shape of the GCLF after subtraction of the background LF is consistent in broad terms with a normal GC population.

At the bright end of the GCLF $(I<23.5)$ a slight excess number of objects is present above the fitted Gaussian curve. Some of these objects could be UCD can- didates, which are known to be present in Perseus in significant numbers (Penny et al. 2011, 2012). These are visible in the CMD of Fig. 7 as a scattering of points above the main GC population. Similar small excess numbers of such objects can be seen in the GCLFs of major galaxies (Harris et al. 2014), and with no additional information to go on, it is difficult to distinguish between very luminous GCs and lower-luminosity UCDs.

\subsection{Color Distribution: Blue and Red Fractions}

In most luminous galaxies the color-index distribution function (CDF) for GCs is roughly bimodal, with "blue" and "red" sequences separated by $\sim 1$ dex in metallicity $[\mathrm{Fe} / \mathrm{H}]$ (e.g. Larsen et al. 2001; Brodie \& Strader 2006; Peng et al. 2006 Harris 2009 Harris et al. 2017c). This is not a universal phenomenon, however, and for the most luminous giants in particular (the Brightest Cluster Galaxies or BCGs), the CDF becomes more uniformly populated (Harris et al. 2016, 2017c). Models for the generation of the ICL and IGCs (cf. the references cited above) further demonstrate that the higher-metallicity GCs should follow a spatial distribution more centrally concentrated to the cluster core, like the member galaxies, whereas the low-metallicity GCs stripped primarily from the dwarfs and outer halos of the member galaxies should follow a more extended distribution closer to the dark-matter potential well. The IGC data from across the Virgo cluster (Durrell et al. 2014) and Coma (Peng et al. 2011, Madrid et al. 2018) are consistent with this prediction.

The CDF from our pairs of outer fields is shown in Figure 14 for all the objects within the GC candidate box, plotted as number versus intrinsic color and in 0.05-mag color bins. Here, we deliberately exclude the data from V11 ACS, because of its proximity to a large galaxy and its obviously higher number density of candidates. The $\mathrm{CDF}$ is also shown for the total numbers of objects in the six control fields, now normalized to the $138 \mathrm{arcmin}^{2}$ area covered by the $(7$ ACS +8 WFC3 $)$ Perseus fields.

The GC candidates show a major, strong peak near $(\mathrm{F} 475 \mathrm{~W}-\mathrm{F} 814 \mathrm{~W})_{0} \simeq 1.5$, which can be identified as the metal-poor GC population. There is a hint of a redder component but it is clearly minor. By comparison, the CDF for the control fields is quite uniform versus color, showing no obvious peaks. Another noteworthy feature is that for $(\mathrm{F} 475 \mathrm{~W}-\mathrm{F} 814 \mathrm{~W})_{0} \gtrsim 2.0$ the numbers of background objects almost precisely match up with the GC candidates.

The difference histogram (GC candidates minus background) is shown in the right panel of Fig. 14. The very reddest objects with $(F 475 W-F 814 W)_{0}>2.1$ have subtracted out well, leaving a statistically pure sample of GCs whose CDF can be matched to a conventional bimodal-Gaussian model to assess the relative fractions of metal-poor or metal-rich clusters. Of the various fitting codes in the recent literature, we use RMIX (e.g. Wehner et al. 2008), which can handle a user-prepared histogram of the CDF. The more frequently used KMM or GMM fitting code (Muratov \& Gnedin 2010), for example, assumes a clean, uncontaminated list of objects (i.e., GCs only) and therefore is less suitable for a dataset containing significant contamination.

RMIX shows that a Gaussian unimodal fit is strongly rejected, at $>99 \%$ significance. For a bimodal fit, the 


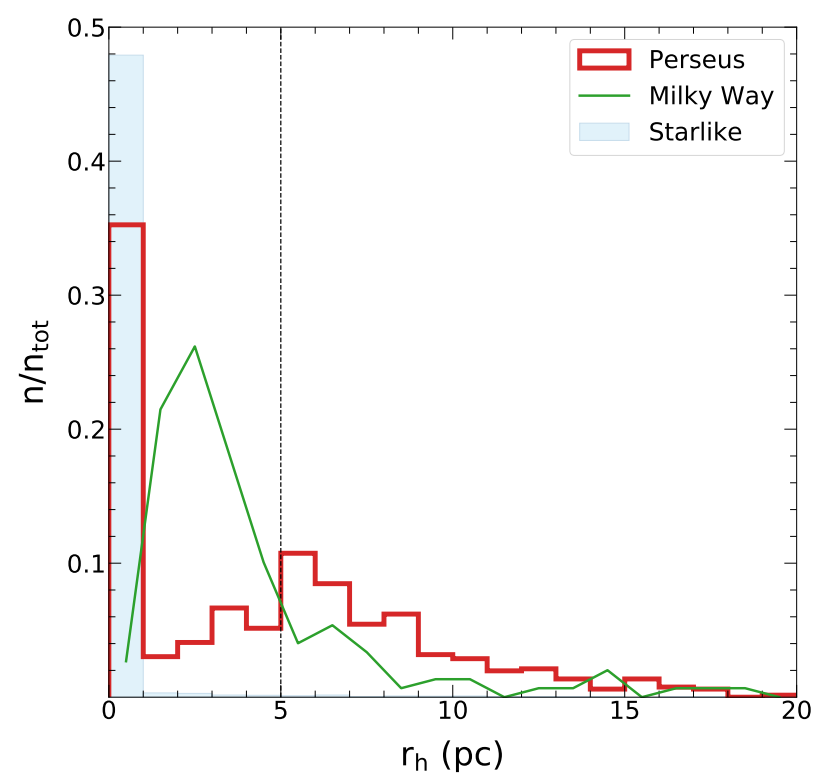

Figure 12. Histogram of intrinsic half-light radius $\left(r_{h}\right.$ or $\left.r_{e f f}\right)$ as deduced from the ISHAPE fitting; $r_{h}$ is shown in parsec units, plotted in 1-pc bins. The Perseus objects in the GC candidate list are shown as the red solid line; simulated starlike objects in the same magnitude range are shown as the cyan shaded histogram; and Milky Way GCs as the solid green line. The vertical line at $r_{h} \simeq 5$ pc indicates a conservative resolution limit above which $r_{h}$ should be measurable at the Perseus distance (see text).

results give best-fit components at colors $\mu_{1}=1.469 \pm$ $0.013, \mu_{2}=1.858 \pm 0.048 ;$ and dispersions $\sigma_{1}=0.160 \pm$ $0.009, \sigma_{2}=0.115 \pm 0.025$. The blue component makes up a fraction $f_{\text {blue }}=0.902 \pm 0.036$ of the total, thus giving $f_{\text {red }}=0.098$. The total fit, shown in Fig. 14, accurately matches the CDF with no clear evidence that any other components are needed within the uncertainties of the data.

We conclude that at least in the outer regions of Perseus, the IGCs are strongly dominated by metalpoor clusters (making up 90\% of the total). Small though it is, however, the red component is significant; the total CDF would not be well fitted without it. A first-order conversion of the color indices to approximate metallicity differences can be made if we combine $\Delta(B-I)=1.222 \Delta(F 475 W-F 814 W)$ with $\Delta(B-I) \simeq 0.375 \Delta[\mathrm{Fe} / \mathrm{H}]$ (Harris 2009), giving $\Delta[\mathrm{Fe} / \mathrm{H}] \simeq 3.26 \Delta(F 475 W-F 814 W)$. The color difference $\mu_{2}-\mu_{1}=(0.389 \pm 0.050)$ thus corresponds to $\Delta[\mathrm{Fe} / \mathrm{H}] \simeq 1.2 \pm 0.1$, similar to what is found in individual large galaxies with well populated MDFs (e.g. Harris 2009 Brodie \& Strader 2006). The dispersion of the blue mode $\sigma_{1}$ corresponds to $\sigma_{1}[\mathrm{Fe} / \mathrm{H}] \simeq 0.49 \pm 0.03$, while the red mode has $\sigma_{2}[\mathrm{Fe} / \mathrm{H}] \simeq 0.37 \pm 0.07$. These dispersions are larger than those in the Milky Way (Harris et al. 2016), which are $\sigma_{1}=0.38$ dex, $\sigma_{2}=0.23$ dex. Part of the difference is likely to be due simply to photometric scatter, but part of it might well be a result of the origin of the IGCs as a composite population drawn from many individual galaxies.

Tracing out where the redder IGCs are located in the various fields shows no detectable concentration towards the known galaxies in the fields. Admittedly, however, such tests are limited by the very small numbers of the red GCs.

We defer additional discussion of this interesting direction till more evidence is available from the CDFs of the major core galaxies. But for the present we suggest that the observed $\mathrm{CDF}$ is consistent with a normal range of GC metallicities.

\subsection{Radial Distribution}

The eight target pairs covering the Perseus IGM that we discuss here have a wide range of radii from the center of Perseus (adopted as the position of NGC 1275, the central $\mathrm{cD}$ galaxy). We can therefore use these to gain a preliminary idea of the projected density of the IGC population versus radius. The present results are shown in Figure 15. Here, the numbers of objects in the GC selection box, after subtraction of the background density level $\phi_{b k g d}=1.33 \operatorname{arcmin}^{-2}$, are plotted as number density versus radius within Perseus, where NGC 1275 is assumed to be at the Perseus center.

For comparison, the outermost fields discussed by HM17 were the shallow WFPC2 images at $R \sim 440 \mathrm{kpc}$, so our present dataset extends almost twice as far out. In Fig. 15 no strong radial dependence is evident; instead, the dominant impression is the field-to-field scatter. HM17 estimated that the IGCs follow a projected density profile $\phi \sim R^{-1.0 \pm 0.4}$, which is also shown in Fig. 15. Within the scatter, a falloff with radius this shallow is consistent with the data and would also be consistent with the shape of the isothermal dark-matter potential well of Perseus as a whole. 


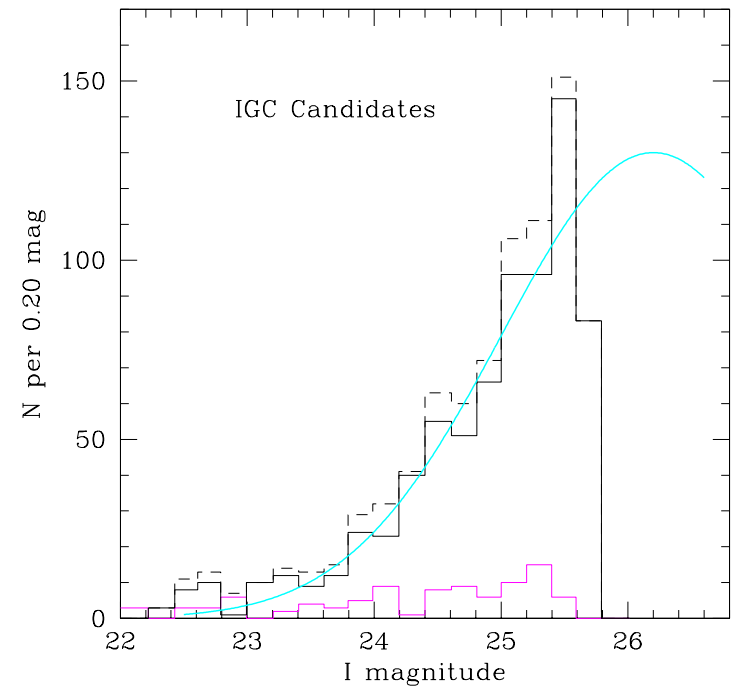

Figure 13. Luminosity distribution of the GC candidates. The dashed line shows the number per 0.2 -mag F $814 \mathrm{~W}$ bin, corrected for incompleteness and extinction $A_{I}$, while the magenta histogram shows the number per bin for the background contamination. The solid histogram shows the difference. The cyan curve shows an illustrative Gaussian luminosity function for $\left(I^{0}, \sigma\right)=(26.2,1.2)$.

If the field-to-field scatter that is quite noticeable in Figs. 8 and 15 is not due strictly to simple stochastic differences in an already-low mean density, part of the explanation may lie in the particular locations of these fields, which are not randomly located across the Perseus region. V12/ACS, V13, and V14/ACS fall along a prominent chain of galaxies running from the Perseus core region (East side) through to IC 310 (the giant ETG on the West side, at lower right in Fig. 1) and beyond. This connection raises the possibility that IGCs may also lie preferentially along the same axis. On a scale an order of magnitude larger, the distribution of Perseus galaxies connects with the Perseus-Pisces supercluster (Haynes \& Giovanelli 1986, Wegner et al. 1993). V11/ACS, V8/ACS, and V15/ACS lie moderately close to major galaxies to the south and northwest. Similarly, the pointings with the lowest number densities (V9, V15/WFC3, V12/WFC3) lie furthest from identifiable substructures. In upcoming work, we will use the Subaru HSC imaging material to gain a more comprehensive picture of the IGCs, including especially their global spatial distribution and their total numbers.

Lastly, it is worth noting that the redder IGCs, though small in number, can be found in even the target fields furthest from the Perseus center. For comparison, Longobardi et al. (2018) found a similar result for IGCs in

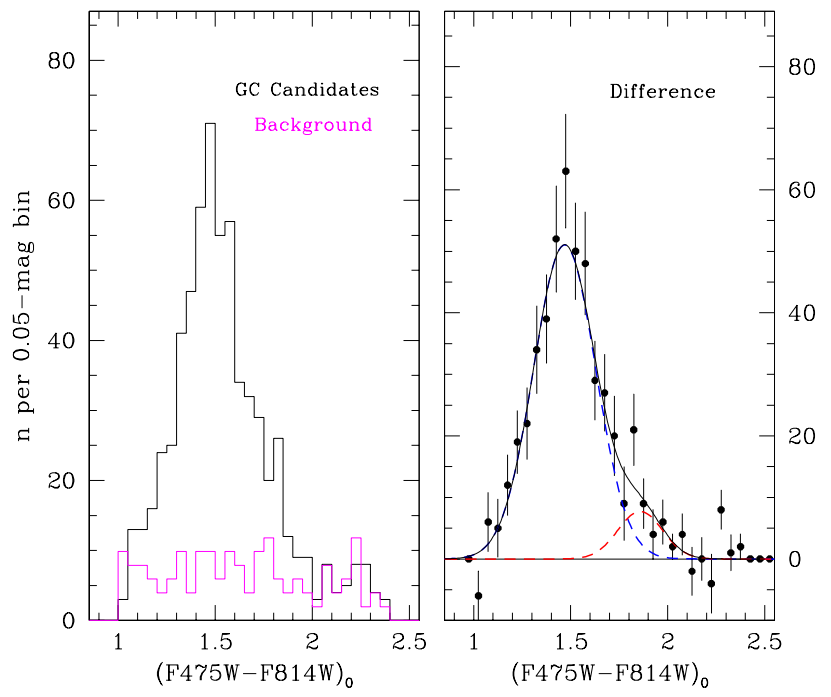

Figure 14. Left panel: Color distribution histograms for the GC candidates in eight pairs of outer fields (in black), and for objects in the six control fields (in magenta). Number of objects per 0.05mag bin is plotted versus intrinsic color $(\mathrm{F} 475 \mathrm{~W}-\mathrm{F} 814 \mathrm{~W})_{0}$. The control-field data are normalized to the same area of $138 \mathrm{arcmin}^{2}$ as covered by the Perseus fields minus V11/ACS (see text). Right panel: Difference between the GC-candidate histogram and the control-field histogram, giving a statistically "clean" color distribution function for the IGCs in Perseus. Errorbars on each data point include the $N^{1 / 2}$ uncertainties in both GC candidates and the background. The bimodal-Gaussian fit to the distribution as described in the text is superimposed as the black line, with the red and blue components shown as dashed lines.

the Virgo cluster: The bluer, lower-metallicity IGCs define a slightly shallower radial distribution than do the higher-metallicity ones, but both components are present to the outermost radii surveyed.

\section{SUMMARY}

We introduce a new imaging program (PIPER) for the giant Perseus cluster of galaxies. The main goals for this program are to survey the globular cluster populations around the giant galaxies in the Perseus core, in 40 UDG candidates, and in the IntraCluster Medium, in addition to building a more comprehensive sample of the UCDs and cEs in Perseus. Upon completion, the program will include photometry in 35 different HST pointings extending from the cluster center (NGC 1275) out to projected radial distances more than $700 \mathrm{kpc}$ from center, as well as Subaru HSC multicolor photometry covering the entire Perseus region to a similar radius.

In the present paper, we outline the photometric reduction methodology for our HST images, and describe the first round of results obtained from eight ACS/WFC3 


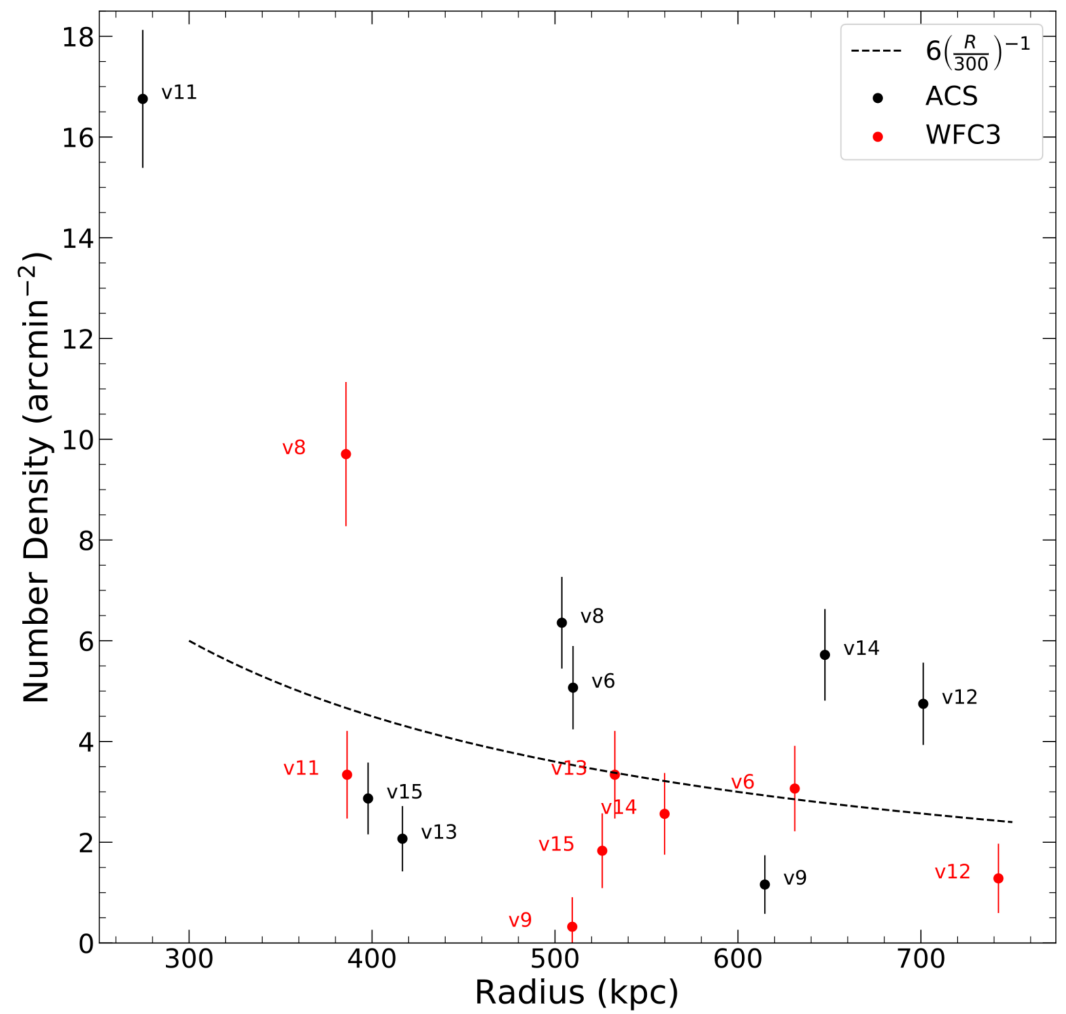

Figure 15. Number density of IGCs in the outer Perseus fields, plotted versus radius from the Perseus center (assumed to be at NGC 1275). Black symbols are ACS fields and red symbols are the WFC3 fields. The magnitude range included here is $22.0<F 814 W_{0}<25.5$. The adopted mean backgrounddensity of 1.33 arcmin $^{-2}$ has been subtracted from all points. Note V11 ACS (upper left) is heavily contaminated by a nearby early-type galaxy. The dashed line shows the $\phi \sim R^{-1}$ radial profile described in the text.

image pairs in the (F475W, F814W) filters (for ACS) and (F475X, F814W) (for WFC3). These HST exposures are designed to be deep enough to approach the LF turnover point of the globular cluster systems in the Perseus galaxies. The eight pairs of fields analyzed here are all located well outside the Perseus core, and we use these to find and characterize the intergalactic globular cluster population (IGC). Field contamination is determined from six Archival HST/ACS fields of similar depth and with similar filters.

The results of this first PIPER study are as follows:

1. The dereddened photometry is used to define a "GC candidate" region in the color-magnitude diagram with boundaries $22.0<F 814 W_{0}<25.5$, $1.0<(F 475 W-F 814 W)_{0}<2.4$. Even at distances $R>700 \mathrm{kpc}$, a sparse but clearly detectable population of objects is present that matches the color and magnitude ranges expected for normal GCs. In almost all of our fields these IGCs dominate over the field contamination.

2. The half-light radii for GCs larger than $r_{h} \gtrsim 5$ pc can be measured (smaller ones are unresolved even with HST). For these, the size distribution resembles that of the Milky Way GCs. A peak near $r_{h} \simeq 5 \mathrm{pc}$ is followed by a long tail extending up to $r_{h} \sim 15 \mathrm{pc}$.

3. The luminosity distribution has the characteristic Gaussian shape in number per unit magnitude, with an estimated turnover point at $I_{0} \simeq 26.2$ $\left(M_{I}=-8.4\right)$ and dispersion $\sigma \simeq 1.2 \mathrm{mag}$.

4. The color distribution is measurably bimodal, but with $90 \%$ of the IGCs belonging to the blue (metalpoor) mode. Conversely, even in these very remote fields a trace population of red (metal-rich) clusters is present, indicating that at least some of the IGC population must have originated in moderately large galaxies.

5. Finally, the distribution of the IGCs with radius from the center of the Perseus cluster is consistent with a shallow $R^{-1}$ falloff, though this trend is obscured by field-to-field scatter.

In future papers of this series, analyses will concentrate on the UDGs and their own GC populations, the central giant galaxies, and the UCDs and compact ellipticals to be discovered in our target fields. Adding in the HSC data from Subaru will also give us a more comprehensive 
assessment of the IGC spatial distribution on its broadest scales.

WEH acknowledges financial support from the Natural Sciences and Engineering Research Council of Canada. AJR was supported by National Science Foundation grants AST-1515084 and 1616710 and as a Research Corporation for Science Advancement Cottrell Scholar. AJR and PRD are supported by funds provided by NASA through grant GO-15235 from the Space Telescope Science Institute, which is operated by the Association of Universities for Research in Astronomy, Incorpo- rated, under NASA contract NAS5-26555. JB acknowledges support from NSF grants AST-1616598 and AST1518294. CW and TL were supported by the Deutsche Forschungsgemeinschaft (DFG, German Research Foundation) through project 394551440. TL also acknowledges financial support from the European Union's Horizon 2020 research and innovation programme under the Marie Skłodowska-Curie grant agreement No. 721463 to the SUNDIAL ITN network. Lastly, we are grateful to the referee for a very constructive review.

\section{HST (ACS, WFC3)}

\section{REFERENCES}

Alamo-Martínez, K. A. \& Blakeslee, J. P. 2017, ApJ, 849, 6 Amorisco, N. C., Monachesi, A., Agnello, A., \& White, S. D. M. 2018, MNRAS, 475, 4235

Beasley, M. A. \& Trujillo, I. 2016, ApJ, 830, 23

Brodie, J. P. \& Strader, J. 2006, ARA\&A, 44, 193

Burke, C., Collins, C. A., Stott, J. P., \& Hilton, M. 2012, MNRAS, 425, 2058

Burkert, A. \& Forbes, D. 2019, arXiv e-prints, arXiv:1901.00900

Canning, R. E. A., Fabian, A. C., Johnstone, R. M., Sanders, J. S., Conselice, C. J., Crawford, C. S., Gallagher, J. S., \& Zweibel, E. 2010, MNRAS, 405, 115

Canning, R. E. A., Ryon, J. E., Gallagher, J. S., Kotulla, R. O'Connell, R. W., Fabian, A. C., Johnstone, R. M., Conselice, C. J., Hicks, A., Rosario, D., \& Wyse, R. F. G. 2014, MNRAS, 444, 336

Carleton, T., Errani, R., Cooper, M., Kaplinghat, M., Peñarrubia, J., \& Guo, Y. 2019, MNRAS, 485, 382

Carlson, M. N., Holtzman, J. A., Watson, A. M., Grillmair, C. J., Mould, J. R., Ballester, G. E., Burrows, C. J., Clarke, J. T., Crisp, D., Evans, R. W., Gallagher, III, J. S., Griffiths, R. E., Hester, J. J., Hoessel, J. G., Scowen, P. A., Stapelfeldt, K. R., Trauger, J. T., \& Westphal, J. A. 1998, AJ, 115, 1778

Chan, T. K., Kereš, D., Wetzel, A., Hopkins, P. F., Faucher-Giguère, C. A., El-Badry, K., Garrison-Kimmel, S., \& Boylan-Kolchin, M. 2018, MNRAS, 478, 906

Colless, M. \& Dunn, A. M. 1996, ApJ, 458, 435

Durrell, P. R., Côté, P., Peng, E. W., Blakeslee, J. P., Ferrarese, L., Mihos, J. C., Puzia, T. H., Lançon, A., Liu, C., Zhang, H., Cuillandre, J.-C., McConnachie, A., Jordán, A., Accetta, K., Boissier, S., Boselli, A., Courteau, S., Duc, P.-A., Emsellem, E., Gwyn, S., Mei, S., \& Taylor, J. E. 2014, ApJ, 794, 103

El-Badry, K., Quataert, E., Weisz, D. R., Choksi, N., \& Boylan-Kolchin, M. 2018, ArXiv e-prints

Fabian, A. C., Sanders, J. S., Allen, S. W., Canning, R. E. A., Churazov, E., Crawford, C. S., Forman, W., Gabany, J., Hlavacek-Larrondo, J., Johnstone, R. M., Russell, H. R., Reynolds, C. S., Salomé, P., Taylor, G. B., \& Young, A. J. 2011, MNRAS, 418, 2154

Ferré-Mateu, A., Forbes, D. A., Romanowsky, A. J., Janz, J., \& Dixon, C. 2018, MNRAS, 473, 1819

Forbes, D. A., Read, J. I., Gieles, M., \& Collins, M. L. M. 2018, MNRAS, 481, 5592

Georgiev, I. Y., Goudfrooij, P., Puzia, T. H., \& Hilker, M. 2008, AJ, 135, 1858

Girardi, L., Groenewegen, M. A. T., Hatziminaoglou, E., \& da Costa, L. 2005, A\&A, 436, 895

Girardi, M., Fadda, D., Giuricin, G., Mardirossian, F., Mezzetti, M., \& Biviano, A. 1996, ApJ, 457, 61

Girardi, M., Giuricin, G., Mardirossian, F., Mezzetti, M., \& Boschin, W. 1998, ApJ, 505, 74

Gómez, M. \& Woodley, K. A. 2007, ApJ, 670, L105

Hanes, D. A. \& Whittaker, D. G. 1987, AJ, 94, 906

Harris, K. A., Debattista, V. P., Governato, F., Thompson, B. B., Clarke, A. J., Quinn, T., Willman, B., Benson, A., Farrah, D., Peng, E. W., Elliott, R., \& Petty, S. 2017a, MNRAS, 467, 4501

Harris, W. E. 1996, AJ, 112, 1487

-. 2009, ApJ, 699, 254

-. 2018, AJ, 156, 296
Harris, W. E., Blakeslee, J. P., \& Harris, G. L. H. 2017b, ApJ, 836,67

Harris, W. E., Blakeslee, J. P., Whitmore, B. C., Gnedin, O. Y., Geisler, D., \& Rothberg, B. 2016, ApJ, 817, 58

Harris, W. E., Ciccone, S. M., Eadie, G. M., Gnedin, O. Y., Geisler, D., Rothberg, B., \& Bailin, J. 2017c, ApJ, 835, 101

Harris, W. E., Morningstar, W., Gnedin, O. Y., O'Halloran, H., Blakeslee, J. P., Whitmore, B. C., Côté, P., Geisler, D., Peng, E. W., Bailin, J., Rothberg, B., Cockcroft, R., \& Barber DeGraaff, R. 2014, ApJ, 797, 128

Harris, W. E. \& Mulholland, C. J. 2017, ApJ, 839, 102

Harris, W. E., Spitler, L. R., Forbes, D. A., \& Bailin, J. 2010, MNRAS, 401, 1965

Haynes, M. P. \& Giovanelli, R. 1986, ApJ, 306, L55

Hinshaw, G., Larson, D., Komatsu, E., Spergel, D. N., Bennett, C. L., Dunkley, J., Nolta, M. R., Halpern, M., Hill, R. S. Odegard, N., Page, L., Smith, K. M., Weiland, J. L., Gold, B. Jarosik, N., Kogut, A., Limon, M., Meyer, S. S., Tucker, G. S., Wollack, E., \& Wright, E. L. 2013, ApJS, 208, 19

Hudson, M. J., Harris, G. L., \& Harris, W. E. 2014, ApJ, 787, L5

Janssens, S., Abraham, R., Brodie, J., Forbes, D., Romanowsky, A. J., \& van Dokkum, P. 2017, ApJ, 839, L17

Janz, J., Norris, M. A., Forbes, D. A., Huxor, A., Romanowsky, A. J., Frank, M. J., Escudero, C. G., Faifer, F. R., Forte, J. C., Kannappan, S. J., Maraston, C., Brodie, J. P., Strader, J., \& Thompson, B. R. 2016, MNRAS, 456, 617

Jiang, F., Dekel, A., Freundlich, J., Romanowsky, A. J., Dutton, A. A., Macciò, A. V., \& Di Cintio, A. 2019, MNRAS, 487, 5272

Jordán, A., Côté, P., Blakeslee, J. P., Ferrarese, L., McLaughlin, D. E., Mei, S., Peng, E. W., Tonry, J. L., Merritt, D.

Milosavljević, M., Sarazin, C. L., Sivakoff, G. R., \& West, M. J. 2005, ApJ, 634, 1002

Jordán, A., McLaughlin, D. E., Côté, P., Ferrarese, L., Peng, E. W., Mei, S., Villegas, D., Merritt, D., Tonry, J. L., \& West, M. J. 2007, ApJS, 171, 101

Kent, S. M. \& Sargent, W. L. W. 1983, AJ, 88, 697

Ko, Y., Hwang, H. S., Lee, M. G., Park, H. S., Lim, S., Sohn, J., Jang, I. S., Hwang, N., \& Park, B.-G. 2017, ApJ, 835, 212

Koda, J., Yagi, M., Yamanoi, H., \& Komiyama, Y. 2015, ApJ, 807, L2

Larsen, S. S. 1999, A\&AS, 139, 393

Larsen, S. S., Brodie, J. P., Huchra, J. P., Forbes, D. A., \& Grillmair, C. J. 2001, AJ, 121, 2974

Lee, M. G., Park, H. S., \& Hwang, H. S. 2010, Science, 328, 334

Lim, J., Wong, E., Ohyama, Y., Broadhurst, T., \& Medezinski, E. 2019, Nature Astronomy, 470

Lim, S., Hwang, N., \& Lee, M. G. 2013, ApJ, 766, 20

Lim, S., Peng, E. W., Cote, P., Sales, L. V., den Brok, M., Blakeslee, J. P., \& Guhathakurta, P. 2018, ArXiv e-prints Loewenstein, M. 1994, ApJ, 431, 91

Longobardi, A., Peng, E. W., Côté, P., Mihos, J. C., Ferrarese, L., Puzia, T. H., Lançon, A., Zhang, H.-X., Muñoz, R. P., Blakeslee, J. P., Guhathakurta, P., Durrell, P. R.,

Sánchez-Janssen, R., Toloba, E., Jordán, A., Eyheramendy, S., Cuilland re, J.-C., Gwyn, S. D. J., Boselli, A., Duc, P.-A., Liu, C., Alamo-Martínez, K., Powalka, M., \& Lim, S. 2018, ApJ, 864,36 
Lotz, J. M., Koekemoer, A., Coe, D., Grogin, N., Capak, P., Mack, J., Anderson, J., Avila, R., Barker, E. A., Borncamp, D., Brammer, G., Durbin, M., Gunning, H., Hilbert, B., Jenkner, H., Khandrika, H., Levay, Z., Lucas, R. A., MacKenty, J., Ogaz, S., Porterfield, B., Reid, N., Robberto, M., Royle, P., Smith, L. J., Storrie-Lombardi, L. J., Sunnquist, B., Surace, J., Taylor, D. C., Williams, R., Bullock, J., Dickinson, M., Finkelstein, S., Natarajan, P., Richard, J., Robertson, B., Tumlinson, J., Zitrin, A., Flanagan, K., Sembach, K., Soifer, B. T., \& Mountain, M. 2017, ApJ, 837, 97

Madrid, J. P., O'Neill, C. R., Gagliano, A. T., \& Marvil, J. R. 2018, ApJ, 867, 144

Main, R. A., McNamara, B. R., Nulsen, P. E. J., Russell, H. R., \& Vantyghem, A. N. 2017, MNRAS, 464, 4360

Martin, G., Kaviraj, S., Laigle, C., Devriendt, J. E. G., Jackson, R. A., Peirani, S., Dubois, Y., Pichon, C., \& Slyz, A. 2019, MNRAS, 485, 796

Martínez-Delgado, D., Läsker, R., Sharina, M., Toloba, E., Fliri, J., Beaton, R., Valls-Gabaud, D., Karachentsev, I. D., Chonis, T. S., Grebel, E. K., Forbes, D. A., Romanowsky, A. J. Gallego-Laborda, J., Teuwen, K., Gómez-Flechoso, M. A., Wang, J., Guhathakurta, P., Kaisin, S., \& Ho, N. 2016, AJ, 151,96

Martinović, N. \& Micic, M. 2017, MNRAS, 470, 4015

Masters, K. L., Jordán, A., Côté, P., Ferrarese, L., Blakeslee, J. P., Infante, L., Peng, E. W., Mei, S., \& West, M. J. 2010 , ApJ, 715, 1419

Matsushita, K., Sakuma, E., Sasaki, T., Sato, K., \& Simionescu, A. 2013, ApJ, 764, 147

Mieske, S., Hilker, M., \& Misgeld, I. 2012, A\&A, 537, A3

Mihos, J. C., Durrell, P. R., Ferrarese, L., Feldmeier, J. J., Côté, P., Peng, E. W., Harding, P., Liu, C., Gwyn, S., \& Cuillandre, J.-C. 2015, ApJ, 809, L21

Muratov, A. L. \& Gnedin, O. Y. 2010, ApJ, 718, 1266

Papastergis, E., Adams, E. A. K., \& Romanowsky, A. J. 2017, A\&A, 601, L10

Peng, E. W., Ferguson, H. C., Goudfrooij, P., Hammer, D., Lucey, J. R., Marzke, R. O., Puzia, T. H., Carter, D., Balcells, M., Bridges, T., Chiboucas, K., del Burgo, C., Graham, A. W. Guzmán, R., Hudson, M. J., Matković, A., Merritt, D., Miller, B. W., Mouhcine, M., Phillipps, S., Sharples, R., Smith, R. J., Tully, B., \& Verdoes Kleijn, G. 2011, ApJ, 730, 23

Peng, E. W., Jordán, A., Côté, P., Blakeslee, J. P., Ferrarese, L., Mei, S., West, M. J., Merritt, D., Milosavljević, M., \& Tonry, J. L. 2006, ApJ, 639, 95

Peng, E. W. \& Lim, S. 2016, ApJ, 822, L31

Penny, S. J., Conselice, C. J., de Rijcke, S., Held, E. V., Gallagher, J. S., \& O'Connell, R. W. 2011, MNRAS, 410, 1076

Penny, S. J., Forbes, D. A., \& Conselice, C. J. 2012, MNRAS, 422, 885

Pfeffer, J., Griffen, B. F., Baumgardt, H., \& Hilker, M. 2014, MNRAS, 444, 3670

Prole, D. J., Hilker, M., van der Burg, R. F. J., Cantiello, M., Venhola, A., Iodice, E., van de Ven, G., Wittmann, C., Peletier, R. F., Mieske, S., Capaccioli, M., Napolitano, N. R., Paolillo, M., Spavone, M., \& Valentijn, E. 2019, MNRAS, 484, 4865
Ramos, F., Coenda, V., Muriel, H., \& Abadi, M. 2015, ApJ, 806, 242

Ramos-Almendares, F., Abadi, M., Muriel, H., \& Coenda, V. 2018, ApJ, 853, 91

Román, J. \& Trujillo, I. 2017, MNRAS, 468, 4039

Rong, Y., Guo, Q., Gao, L., Liao, S., Xie, L., Puzia, T. H., Sun, S., \& Pan, J. 2017, MNRAS, 470, 4231

Simionescu, A., Allen, S. W., Mantz, A., Werner, N., Takei, Y., Morris, R. G., Fabian, A. C., Sanders, J. S., Nulsen, P. E. J., George, M. R., \& Taylor, G. B. 2011, Science, 331, 1576 Sirianni, M., Jee, M. J., Benítez, N., Blakeslee, J. P., Martel, A. R., Meurer, G., Clampin, M., De Marchi, G., Ford, H. C., Gilliland, R., Hartig, G. F., Illingworth, G. D., Mack, J., \& McCann, W. J. 2005, PASP, 117, 1049

Struble, M. F. \& Rood, H. J. 1991, ApJS, 77, 363

Toloba, E., Lim, S., Peng, E., Sales, L. V., Guhathakurta, P., Mihos, J. C., Côté, P., Boselli, A., Cuillandre, J.-C., Ferrarese, L., Gwyn, S., Lançon, A., Muñoz, R., \& Puzia, T. 2018, ApJ, 856, L31

van Dokkum, P., Abraham, R., Brodie, J., Conroy, C., Danieli, S., Merritt, A., Mowla, L., Romanowsky, A., \& Zhang, J. 2016, ApJ, 828, L6

van Dokkum, P., Cohen, Y., Danieli, S., Kruijssen, J. M. D., Romanowsky, A. J., Merritt, A., Abraham, R., Brodie, J.,

Conroy, C., Lokhorst, D., Mowla, L., O'Sullivan, E., \& Zhang, J. 2018, ApJ, 856, L30

van Dokkum, P. G., Abraham, R., Merritt, A., Zhang, J., Geha, M., \& Conroy, C. 2015a, ApJ, 798, L45

van Dokkum, P. G., Romanowsky, A. J., Abraham, R., Brodie, J. P., Conroy, C., Geha, M., Merritt, A., Villaume, A., \& Zhang, J. 2015b, ApJ, 804, L26

Villegas, D., Jordán, A., Peng, E. W., Blakeslee, J. P., Côté, P., Ferrarese, L., Kissler-Patig, M., Mei, S., Infante, L., Tonry, J. L., \& West, M. J. 2010, ApJ, 717, 603

Voggel, K. T., Seth, A. C., Neumayer, N., Mieske, S., Chilingarian, I., Ahn, C., Baumgardt, H., Hilker, M., Nguyen, D. D., Romanowsky, A. J., Walsh, J. L., den Brok, M., \& Strader, J. 2018, ApJ, 858, 20

Wegner, G., Haynes, M. P., \& Giovanelli, R. 1993, AJ, 105, 1251

Wehner, E. M. H., Harris, W. E., Whitmore, B. C., Rothberg, B., \& Woodley, K. A. 2008, ApJ, 681, 1233

Weinmann, S. M., Lisker, T., Guo, Q., Meyer, H. T., \& Janz, J. 2011, MNRAS, 416, 1197

West, M. J., Jordán, A., Blakeslee, J. P., Côté, P., Gregg, M. D., Takamiya, M., \& Marzke, R. O. 2011, A\&A, 528, A115

Williams, B. F., Ciardullo, R., Durrell, P. R., Feldmeier, J. J., Sigurdsson, S., Vinciguerra, M., Jacoby, G. H., von Hippel, T., Ferguson, H. C., Tanvir, N. R., Arnaboldi, M., Gerhard, O., Aguerri, J. A. L., \& Freeman, K. C. 2007, ApJ, 654, 835

Wittmann, C., Lisker, T., Ambachew Tilahun, L., Grebel, E. K., Conselice, C. J., Penny, S., Janz, J., Gallagher, J. S., Kotulla, R., \& McCormac, J. 2017, MNRAS, 470, 1512

Wittmann, C., Lisker, T., Pasquali, A., Hilker, M., \& Grebel, E. K. 2016, MNRAS, 459, 4450

Zhang, Y. \& Bell, E. F. 2017, ApJ, 835, L2

Zhao, H.-H., Jia, S.-M., Chen, Y., Li, C.-K., Song, L.-M., \& Xie, F. 2013, ApJ, 778, 124 